Research Article

\title{
Further Developments of Bessel Functions via Conformable Calculus with Applications
}

\author{
Mahmoud Abul-Ez $\mathbb{D}^{1}$, Mohra Zayed $\mathbb{D}^{2},{ }^{2}$ and Ali Youssef $\mathbb{D}^{1}$ \\ ${ }^{1}$ Mathematics Department, Faculty of Science, Sohag University, Sohag 82524, Egypt \\ ${ }^{2}$ Mathematics Department, College of Science, King Khalid University, Abha, Saudi Arabia \\ Correspondence should be addressed to Mohra Zayed; mzayed@kku.edu.sa
}

Received 9 June 2021; Revised 1 August 2021; Accepted 26 August 2021; Published 27 September 2021

Academic Editor: Badr Saad. T. Alkaltani

Copyright (c) 2021 Mahmoud Abul-Ez et al. This is an open access article distributed under the Creative Commons Attribution License, which permits unrestricted use, distribution, and reproduction in any medium, provided the original work is properly cited.

\begin{abstract}
The theory of Bessel functions is a rich subject due to its essential role in providing solutions for differential equations associated with many applications. As fractional calculus has become an efficient and successful tool for analyzing various mathematical and physical problems, the so-called fractional Bessel functions were introduced and studied from different viewpoints. This paper is primarily devoted to the study of developing two aspects. The starting point is to present a fractional Laplace transform via conformable fractional-order Bessel functions (CFBFs). We establish several important formulas of the fractional Laplace Integral operator acting on the CFBFs of the first kind. With this in hand, we discuss the solutions of a generalized class of fractional kinetic equations associated with the CFBFs in view of our proposed fractional Laplace transform. Next, we derive an orthogonality relation of the CFBFs, which enables us to study an expansion of any analytic functions by means of CFBFs and to propose truncated CFBFs. A new approximate formula of conformable fractional derivative based on CFBFs is provided. Furthermore, we describe a useful scheme involving the collocation method to solve some conformable fractional linear (nonlinear) multiorder differential equations. Accordingly, several practical test problems are treated to illustrate the validity and utility of the proposed techniques and examine their approximate and exact solutions. The obtained solutions of some fractional differential equations improve the analog ones provided by various authors using different techniques. The provided algorithm may be beneficial to enrich the Bessel function theory via fractional calculus.
\end{abstract}

\section{Introduction}

The theory of special functions is a critical branch of modern mathematical analysis. During the past three decades, several new classes of special functions have been proposed as solutions of fractional differential equations (FDEs). No other special functions have received such detailed treatment in readily available treatises as Bessel functions. The investigation of such functions is an important problem in fractional calculus, which has earned much attention as real-life problems can be analyzed well. Fractional calculus appears in many branches of science, such as medicine, material sciences, electromagnetics, and fluid mechanics (see [1-4]). Many applications have been performed through FDEs, and their solution techniques could be found, for example, in [5-11].
We trace the existing efforts regarding fractional order derivatives. Several definitions of the fractional order derivative have been introduced by many famous authors, such as Euler, Fourier, Letnikov, Laurent, Grünwald-Letnikov, Caputo, and Riemann-Liouville. Other definitions have also been provided by Kilbas et al. and Miller and Ross in $[3,10]$. The most popular definitions considered frequently in the literature are derivatives by Riemann-Liouville, Caputo, and Grünwald-Letnikov. Interestingly, each definition of the arbitrary order derivative captures only a few properties of the classical integral derivative. However, a few drawbacks exist; for instance, $\mathscr{D}_{a}^{\alpha}(1)=0$ does not fulfill the RiemannLiouville definition. In Caputo's definition, $f(x)$ is assumed to be differentiable; otherwise, one cannot use such a definition. Moreover, Liouville's theorem in the fractional setting does not hold. Therefore, it is clear that all definitions of 
fractional derivatives seem deficient regarding certain mathematical properties, such as the rules of product, quotient, and chain. For more details concerning other properties of these fractional order derivatives, see, for example, [4] and the references therein.

Due to the mentioned arguments, a new definition of the fractional order derivative is needed to achieve suitable mathematical properties. Khalil et al. [12] introduced a well-extended definition of the noninteger order derivative called the conformable fractional derivative (CFD). This definition is formulated as follows:

Definition 1 (see [12]). For the initial real value $a$, the conformable fractional derivative $\mathscr{D}_{a}^{\alpha} f(x)$ of a real function $f:[a, \infty) \longrightarrow \mathbb{R}, \alpha \in(0,1]$ is defined by the following:

$$
\mathscr{D}_{a}^{\alpha} f(x)=\lim _{h \longrightarrow 0} \frac{f\left(x+h(x-a)^{1-\alpha}\right)-f(x)}{h}, \text { for all } x>a
$$

The initial value $a$ can be zero, and if the limit exists, then $f(x)$ is called $\alpha$-differentiable.

Along with the CFD's Definition 1, if $f(x)$ is differentiable, then $\mathscr{D}^{\alpha} f(x)=x^{1-\alpha} f^{\prime}(x)$ (see [12]). Moreover, if Definition 1 holds for $\alpha=0$, we obtain $\mathscr{D}^{0} f(x)=f(x)$. Additionally, we have $\mathscr{D}^{0} f(x)=x f^{\prime}(x)$; hence, $x=f(x) / f^{\prime}(x)$, which indicates that $x$ relies on some functions; it is unreasonable. Therefore, the CFD definition [12] does not need to hold for zero order. For the conformable fractional integral, we state the following definition as given in the following [12]:

Definition 2 (see [12]). Let $f:[0, \infty) \longrightarrow \mathbb{R}$. Then, for any $\beta \in(0,1]$, the conformable fractional integral $I_{\beta} f(x)$ of order $\beta$ of $f$ is defined as follows:

$$
I_{\beta} f(x)=\int_{0}^{x} t^{\beta-1} f(t) d t
$$

Definition 1 depends entirely on the basic limit like the classical order derivative. Furthermore, Definition 1 fulfills various classical properties, such as the mean value theorem and the product, quotient, and chain rules. Moreover, this definition is provided with the Leibniz rule, in which other fractional derivatives can not achieve (see [13]). Growing attention has been paid to explore the conformable derivatives due to the enormous number of their meaningful applications in many fields of science. Abul-Ez et al. [14] introduced a comprehensive study on the conformable fractional Legendre polynomials. They presented the shifted conformable fractional Legendre polynomials and described an applicable scheme using the collocation method to solve some fractional differential equations (FDEs) in the sense of conformable derivative. Recently, the conformable fractional Gauss hypergeometric function and a class of conformable fractional differential equations through that function were treated in [15]. Further interesting ideas on the conformable derivative can be found in the work by [16-23].
Note that some authors have demonstrated that the conformable derivative is not the same as a fractional order derivative, but it is a first-order derivative multiplied by an additional factor (see for example [23]). Hence, Definition 1 seems to be a natural extension of the conventional order derivative to noninteger order loosing memory effect. In addition, a new approach for finding fractional operators was introduced by Antagan and Baleanu [24] with a nonsingular Mittag-Leffler kernel with a memory effect.

Returning to the purpose of the present work, we observe that Bessel functions are playing a significant role in investigating the solution of important differential equations (for example, see [25]). The theory of Bessel functions is usually used when solving problems related to information theory, nuclear physics, radiophysics, and hydrodynamics. Recently, as in [26-30], a resurgence of interest has occurred in the study of Bessel functions in the framework of fractional calculus theory. Along with the work in [26, 27], we employ conformable fractional order Bessel functions (CFBFs) to solve problems of a fractional nature. The study of a Bessel function of half-integer order led to discovering another interesting class of orthogonal polynomials called the Bessel polynomials. Many authors have used these polynomials. For example, Yüzbaşi et al. [31] solved linear integral, differential, and integro-differential equations, while Parand et al. [32] applied Bessel functions to solve nonlinear Lane-Emden equations. In [33], fractional optimal control problems were solved using the Bessel collocation method.

The present work proposes an approach to approximating the solution for some important linear and nonlinear FDEs in the conformable sense. The paper is designed with two objectives. The first is to establish some interesting properties of fractional Laplace-type integrals of functions via CFBFs. Then, we use the obtained results to establish the possible solutions of conformable fractional kinetic equations through CFBFs. The second objective is concerned with developing applications of the fundamental process of the proposed approach in terms of CFBFs. To achieve that, we derive an orthogonality relation, expand functions in terms of the truncated CFBFs, and effectively formulate a scheme involving the collocation method which employed to provide solutions of certain types of linear and nonlinear CFBEs.

The structure of this paper is organized as follows. The needed concepts and features of CFD are collected in Section 2. Next, Section 3 establishes useful properties of Laplace transforms in the sense of CFBFs, with some applications to solve a new type of conformable fractional kinetic equations. Section 4 is divided into four subsections. Section 4.1 provides essential results on orthogonality relations. A brief study on an expansion of any analytic function employing CFBFs is the subject of Section 4.2. In Section 4.3 , we construct an algorithm for solving various kinds of problems using CFBFs through the collocation method. Section 4.4 presents the concepts that have been developed through previous subsections to solve some linear and nonlinear conformable fractional differential equations (CFDEs), including the nonlinear Riccati FDE. Concluding remarks are provided in Section 5. 


\section{Preliminaries and Basic Concepts}

The Bessel equation is a special case of the Sturm-Liouville problem, and it can be written as [34]

$$
x^{2} y^{\prime \prime}+x y^{\prime}+\left(x^{2}-n^{2}\right) y=0 .
$$

In view of formula (1), the authors of [26] solved the following conformable fractional Bessel equation:

$$
x^{2 \alpha} \mathscr{D}^{\alpha} \mathscr{D}^{\alpha} y+\alpha x^{\alpha} \mathscr{D}^{\alpha} y+\alpha^{2}\left(x^{2 \alpha}-n^{2}\right) y=0,
$$

around the regular singular point $x=0$, and introduced the CFBFs of the first kind $J_{n}^{\alpha}(x)$ as its solution such that

$$
J_{n}^{\alpha}(x)=\sum_{\kappa=0}^{\infty} \frac{(-1)^{\kappa}}{\kappa ! \Gamma(n+\kappa+1)}\left(\frac{x^{\alpha}}{2}\right)^{n+2 \kappa}
$$

Moreover, they investigated in [26] some of its recurrence relations from which we may mention

$$
\begin{aligned}
\mathscr{D}^{\alpha}\left[x^{\alpha n} J_{n}^{\alpha}(x)\right] & =\alpha x^{\alpha n} J_{n-1}^{\alpha}(x), \\
\mathscr{D}^{\alpha}\left[x^{-\alpha n} J_{n}^{\alpha}(x)\right] & =-\alpha x^{-\alpha n} J_{n+1}^{\alpha}(x), \\
\mathscr{D}^{\alpha}\left[J_{n}^{\alpha}(x)\right] & =\alpha J_{n-1}^{\alpha}(x)-\frac{\alpha n}{x^{\alpha}} J_{n}^{\alpha}(x), \\
\mathscr{D}^{\alpha}\left[J_{n}^{\alpha}(x)\right] & =\frac{\alpha n}{x^{\alpha}} J_{n}^{\alpha}(x)-\alpha J_{n+1}^{\alpha}(x),
\end{aligned}
$$

In the following, we are about to recall some essential definitions and results which are needed in the sequel.

Definition 3. The Gauss hypergeometric function ${ }_{2} F_{1}(a, b$; $c ; x)$ is defined by (see [35])

$$
{ }_{2} F_{1}(a, b ; c ; x)=\sum_{n=0}^{\infty} \frac{(a)_{n}(b)_{n}}{(c)_{n}} \frac{x^{n}}{n !}, \quad|x|<1
$$

where $(\delta)_{n}$ stands for the familiar Pochhammar symbol which can be written in terms of Gamma function as

$(\delta)_{n}=\frac{\Gamma(\delta+n)}{\Gamma(\delta)}=\delta(\delta+1)(\delta+2), \cdots,(\delta+n-1), n \in \mathbb{N},(\delta)_{0}=1$

Definition 4 (see [36]). The function ${ }_{p} \psi_{q}(x)$ where $p$ and $q$ refer to its numerators and denominators, respectively, is called the Fox-Wright function, and it can be defined by the formula

$$
{ }_{p} \psi_{q}(x)={ }_{p} \psi_{q}\left(\begin{array}{l}
\left(a_{i}, \mu_{i}\right)_{1, p} \\
\left(b_{j}, v_{j}\right)_{1, q}
\end{array} ; x\right)=\sum_{n=0}^{\infty} \frac{\prod_{i=1}^{p} \Gamma\left(a_{i}+n \mu_{i}\right)}{\prod_{j=1}^{q} \Gamma\left(b_{j}+n v_{j}\right)} \frac{x^{n}}{n !},
$$

such that $\sum_{j=1}^{q} v_{j}-\sum_{i=1}^{p} \mu_{i}>-1$, where $a_{i}, b_{j} \in \mathbb{R}(i=1,2, \cdots$, $p ; j=1,2, \cdots, q)$.

In particular, when $\mu_{i}=v_{j}=1$ in Definition 4 , then the function ${ }_{p} \psi_{q}(x)$ immediately reduced to the generalized hypergeometric function ${ }_{p} F_{q}$ (see [35]). Abdeljawad [16] defined the fractional Laplace transform in the conformable sense as follows:

Definition 5 (see [16]). For a real valued function $f:[0, \infty)$ $\longrightarrow \mathbb{R}$, the conformable fractional Laplace transform of noninteger order $\alpha, \alpha \in(0,1]$ is given by

$$
L_{\alpha}[f(t)]=F_{\alpha}(s)=\int_{0}^{\infty} e^{-s\left(t^{\alpha} / \alpha\right)} f(t) d_{\alpha} t=\int_{0}^{\infty} e^{-s\left(t^{\alpha} / \alpha\right)} f(t) t^{\alpha-1} d t
$$

The inverse fractional Laplace transform is the transformation of a fractional Laplace transform into a function of time. If $L_{\alpha}[f(t)]=F_{\alpha}(s)$, then $f(t)$ is the inverse fractional Laplace transform of $F_{\alpha}(s)$, and it can be written as

$$
L_{\alpha}^{-1}\left[F_{\alpha}(s)\right]=f(t) .
$$

Remark 6. If $\alpha=1$, then (10) is the classical definition of the Laplace transform of integer order.

Furthermore, the author in [16] gave the following interesting results.

Lemma 7 [16]. For a real valued function $f:[0, \infty) \longrightarrow \mathbb{R}$ satisfying $L_{\alpha}[f(t)]=F_{\alpha}(s), \alpha \in(0,1]$, the following relations hold true:

$$
\begin{aligned}
& \text { (1) } F_{\alpha}(s)=L\left[f(\alpha t)^{1 / \alpha}\right] \text {, where } L[f(t)]=\int_{0}^{\infty} e^{-s t} f(t) d t \\
& \text { (2) } L_{\alpha}[1]=1 / s, s>0 \\
& \text { (3) } L_{\alpha}\left[t^{p}\right]=\alpha^{p / \alpha}\left((\Gamma(1+(p / \alpha))) /\left(s^{1+(p / \alpha)}\right)\right), s>0 \\
& \text { (4) } L_{\alpha}\left[e^{k\left(t^{\alpha} / \alpha\right)}\right]=1 /(s-k)
\end{aligned}
$$

\section{Fractional Laplace Transform of the CFBFs}

1 In this section, we derive some new interesting fractional Laplace-type integrals of functions involving CFBFs. Then, as an application, we are going to employ the obtained results in order to find the possible solutions of the fractional kinetic equations in the conformable sense associated with CFBFs.

\subsection{Fractional Laplace Integral Formulas}

Theorem 8. Let $J_{n}^{\alpha}(x), \alpha \in(0,1]$ be the CFBFs; then,

$$
L_{\alpha}\left\{J_{n}^{\alpha}(t)\right\}=\frac{\alpha^{n}}{2^{n} s^{n+1}}{ }_{2} F_{1}\left(\frac{n+1}{2}, \frac{n}{2}+1 ; n+1 ; \frac{-\alpha^{2}}{s^{2}}\right) .
$$


Proof. Owing to the definition of CFBFs (5) and applying the conformable fractional Laplace transform operator of order $\alpha \in(0,1]$ as stated in Lemma 7 , we have

$$
L_{\alpha}\left\{J_{n}^{\alpha}(t)\right\}=\sum_{\kappa=0}^{\infty} \frac{(-1)^{\kappa}}{\kappa ! \Gamma(n+\kappa+1) 2^{n+2 \kappa}} L_{\alpha}\left\{t^{\alpha(n+2 \kappa)}\right\} .
$$

According to (3) of Lemma 7, one can see

$$
\begin{aligned}
L_{\alpha}\left\{J_{n}^{\alpha}(t)\right\} & =\sum_{\kappa=0}^{\infty} \frac{(-1)^{\kappa}}{\kappa ! \Gamma(n+\kappa+1) 2^{n+2 \kappa}} \frac{\alpha^{n+2 \kappa} \Gamma(n+2 \kappa+1)}{s^{n+2 \kappa+1}} \\
& =\frac{\alpha^{n}}{2^{n} s^{n+1}} \sum_{\kappa=0}^{\infty} \frac{\Gamma(n+2 \kappa+1)}{\kappa ! \Gamma(n+\kappa+1) 2^{2 \kappa}}\left[\frac{-\alpha^{2}}{s^{2}}\right]^{\kappa} \\
& =\frac{\alpha^{n}}{2^{n} s^{n+1}} \sum_{\kappa=0}^{\infty} \frac{(n+1)_{2 \kappa}}{\kappa !(n+1)_{\kappa} 2^{2 \kappa}}\left[\frac{-\alpha^{2}}{s^{2}}\right]^{\kappa} .
\end{aligned}
$$

Using the identity $(n+1)_{2 \kappa}=2^{2 \kappa}((n+1) / 2)_{\kappa}((n / 2)+1)_{\kappa}$, we obtain

$$
L_{\alpha}\left\{J_{n}^{\alpha}(t)\right\}=\frac{\alpha^{n}}{2^{n} s^{n+1}} \sum_{\kappa=0}^{\infty} \frac{((n+1) / 2)_{\kappa}((n / 2)+1)_{\kappa}}{\kappa !(n+1)_{\kappa}}\left[\frac{-\alpha^{2}}{s^{2}}\right]^{\kappa} .
$$

Therefore, the result is established.

Now, consider the Fox-Wright function ${ }_{1} \Psi_{1}$ defined in (9) to deduce the following important results.

Theorem 9. Let $J_{n}^{\alpha}(x), \alpha \in(0,1]$ be the CFBFs. Then, the following relation is satisfied:

$$
L_{\alpha}\left\{J_{n}^{\alpha}\left(a^{\mu} t^{\mu}\right)\right\}=\frac{(\alpha a)^{\mu n}}{2^{n} s^{\mu n+1}}{ }_{1} \psi_{1}\left(\begin{array}{c}
(\mu n+1,2 \mu) \\
(n+1,1)
\end{array} ; \frac{(\alpha a)^{2 \mu}}{4 s^{2 \mu}}\right) .
$$

Proof. By combining (5) and (10), we get

$$
\begin{aligned}
L_{\alpha}\left\{J_{n}^{\alpha}\left(a^{\mu} t^{\mu}\right)\right\} & =L_{\alpha}\left\{\sum_{\kappa=0}^{\infty} \frac{(-1)^{\kappa}}{\kappa ! \Gamma(n+\kappa+1)}\left(\frac{a^{\mu} t^{\mu \alpha}}{2}\right)^{(n+2 \kappa)}\right\} \\
& =\sum_{\kappa=0}^{\infty} \frac{(-1)^{\kappa} a^{\mu(n+2 \kappa)}}{\kappa ! \Gamma(n+\kappa+1) 2^{n+2 \kappa}} L_{\alpha}\left\{t^{\alpha \mu(n+2 \kappa)}\right\} .
\end{aligned}
$$

Due to (3) of Lemma 7, it follows that

$$
\begin{aligned}
L_{\alpha}\left\{J_{n}^{\alpha}\left(a^{\mu} t^{\mu}\right)\right\}= & \sum_{\kappa=0}^{\infty} \frac{(-1)^{\kappa} a^{\mu(n+2 \kappa)}}{\kappa ! \Gamma(n+\kappa+1) 2^{n+2 \kappa}} \\
& \cdot \frac{\alpha^{\mu(n+2 \kappa)} \Gamma(\mu n+2 \mu \kappa+1)}{s^{\mu n+2 \mu \kappa+1}} \\
= & \frac{\alpha^{\mu n} a^{\mu n}}{2^{n} s^{\mu n+1}} \sum_{\kappa=0}^{\infty} \frac{\Gamma(\mu n+2 \mu \kappa+1)}{\kappa ! \Gamma(n+\kappa+1)}\left[\frac{-(\alpha a)^{2 \mu}}{4 s^{2 \mu}}\right]^{\kappa} \\
= & \frac{(\alpha a)^{\mu n}}{2^{n} s^{\mu n+1}}{ }_{1} \psi_{1}\left(\begin{array}{c}
(\mu n+1,2 \mu) \\
(n+1,1)
\end{array} ; \frac{-(\alpha a)^{2 \mu}}{4 s^{2 \mu}}\right),
\end{aligned}
$$

as required.

Theorem 10. Let $J_{n}^{\alpha}(x), \alpha \in(0,1]$ be the CFBFs. Then,

$$
L_{\alpha}\left\{t^{\alpha(\mu-1)} J_{n}^{\alpha}(t)\right\}=\frac{(\alpha)^{n+\mu}}{2^{n} s^{n+\mu} 1} \psi_{1}\left(\begin{array}{c}
(n+\mu, 2) \\
(n+1,1)
\end{array} ; \frac{-\alpha^{2}}{4 s^{2}}\right) .
$$

Proof. As proceeded in the proof of Theorem 8 then, relying on Equation (5) and Lemma 7, we conclude that

$$
\begin{aligned}
L_{\alpha}\left\{t^{\alpha(\mu-1)} J_{n}^{\alpha}(t)\right\}= & \sum_{\kappa=0}^{\infty} \frac{(-1)^{\kappa}}{\kappa ! \Gamma(n+\kappa+1) 2^{n+2 \kappa}} L_{\alpha}\left\{t^{\alpha(n+2 \kappa+\mu-1)}\right\} \\
= & \sum_{\kappa=0}^{\infty} \frac{(-1)^{\kappa}}{\kappa ! \Gamma(n+\kappa+1) 2^{n+2 \kappa}} \\
& \cdot \frac{\alpha^{(n+2 \kappa+\mu)} \Gamma(n+2 \kappa+\mu)}{s^{(n+2 \kappa+\mu)}} \\
= & \frac{\alpha^{n+\mu}}{2^{n} s^{n+\mu}} \sum_{\kappa=0}^{\infty} \frac{\Gamma(n+2 \kappa+\mu)}{\Gamma(n+\kappa+1) \kappa !}\left(\frac{-\alpha^{2}}{4 s^{2}}\right)^{\kappa} .
\end{aligned}
$$

Theorem 11. Let $J_{n}^{\alpha}(x), \alpha \in(0,1]$ be the CFBFs. Then, the following identity holds:

$$
L_{\alpha}\left\{t^{\alpha(\mu-1)} J_{n}^{\alpha}\left(\frac{1}{t}\right)\right\}=\frac{(\alpha)^{\mu-n}}{2^{n} s^{\mu-n}{ }_{1}} \psi_{1}\left(\begin{array}{c}
(\mu-n,-2) \\
(n+1,1)
\end{array} ; \frac{-s^{2}}{4 \alpha^{2}}\right) .
$$


Proof. The reduction of (5) and (10) yields

$$
\begin{aligned}
L_{\alpha}\left\{t^{\alpha(\mu-1)} J_{n}^{\alpha}\left(\frac{1}{t}\right)\right\}= & \sum_{\kappa=0}^{\infty} \frac{(-1)^{\kappa}}{\kappa ! \Gamma(n+\kappa+1) 2^{n+2 \kappa}} L_{\alpha}\left\{t^{\alpha(\mu-n-2 \kappa-1)}\right\} \\
= & \sum_{\kappa=0}^{\infty} \frac{(-1)^{\kappa}}{\kappa ! \Gamma(n+\kappa+1) 2^{n+2 \kappa}} \\
& \cdot \frac{\alpha^{(\mu-n-2 \kappa)} \Gamma(\mu-n-2 \kappa)}{s^{(\mu-n-2 \kappa)}} \\
= & \frac{\alpha^{\mu-n}}{2^{n} s^{\mu-n}} \sum_{\kappa=0}^{\infty} \frac{\Gamma(\mu-n-2 \kappa)}{\Gamma(n+\kappa+1) \kappa !}\left(\frac{-s^{2}}{4 \alpha^{2}}\right)^{\kappa},
\end{aligned}
$$

which ends the proof.

The above obtained results provide the necessary tools which enable us to carry out the following interesting study.

3.2. Fractional Kinetic Equations Associated with the CFBFs and Their Solutions via Fractional Laplace Transform. One of the most important equations in mathematical physics and natural sciences is the kinetic equation, which describes the continuity of motion of substances. Therefore, many researchers investigated extensions and generalizations of this equation in the context of various fractional calculus operators. For such type of work, we refer for example to $[37,38]$. We begin by briefly reviewing these previous efforts; then, we introduce our extended form of the fractional kinetic differential equation associated with the CFBFs. Assuming that $\mathcal{N}(t)$ denotes an arbitrary reaction which depends on time, $d$ refers to the destruction rate, and $p$ is the production rate on $\mathcal{N}$, Haubold and Mathai [37] characterized the FDE of the quantities $\mathcal{N}(t), d$ and $p$, by the formula

$$
\frac{d \mathcal{N}}{d t}=-d\left(\mathcal{N}_{t}\right)+p\left(\mathcal{N}_{t}\right)
$$

where $\mathcal{N}_{t}\left(t^{*}\right)=\mathcal{N}\left(t-t^{*}\right)$ for $t^{*}>0$. In the case where spatial fluctuation or inhomogeneities in the quantity $\mathcal{N}(t)$ is neglected, the authors in [37] handled the following equation:

$$
\frac{d \mathcal{N}_{i}}{d t}=-c_{i} \mathcal{N}_{i}(t)
$$

where the primary condition $\mathcal{N}_{i}(t=0)=\mathcal{N}_{0}$ gives the number density of species $i$ at time $t=0$ and constant $c_{i}>0$. Equation (24) is known as the standard kinetic equation. Alternatively, if the index $i$ is neglected, then, by integrating the standard kinetic Equation (24), one can get

$$
\mathcal{N}(t)-\mathcal{N}_{0}=c_{0} \mathscr{D}^{-1} \mathcal{N}(t)
$$

where ${ }_{0} \mathscr{D}^{-1}$ is the standard integral operator. Equation Equation (25) has been extended to the fractional setting in the form (see [37]).

$$
\mathcal{N}(t)-\mathcal{N}_{0}=c_{0} \mathscr{D}^{-v} \mathcal{N}(t)
$$

where ${ }_{0} \mathscr{D}^{-v}$ denotes the standard fractional integral operator in Riemann-Liouville sense (see [10,39]).

Now, consider the conformable fractional kinetic equation in the form:

$$
\mathcal{N}(t)-\mathcal{N}_{0}=c I_{\alpha}(\mathcal{N}(t))
$$

where $I_{\alpha}(\cdot)$ is the conformable fractional integral of order $v \in(0,1]$ in the frame of Definition 2. Accordingly, we develop here a new type of generalization of the fractional kinetic differential equation in the conformable sense involving the fractional-order Bessel function in view of a fractional Laplace transform.

Remark 12. The solutions we are going to conclude for the conformable fractional kinetic equations will be determined through the generalized Mittag-Leffler function $E_{\mu, v}(x)$ [36], which is defined as

$$
E_{\mu, v}(x)=\sum_{n=0}^{\infty} \frac{x^{n}}{\Gamma(\mu n+v)}, \quad \mu, v>0 .
$$

Theorem 13. For $d>0$ and $\alpha \in(0,1]$, the following conformable fractional equation

$$
\mathcal{N}(t)-\mathcal{N}_{0}\left\{J_{n}^{\alpha}(t)\right\}=-d^{\alpha} I_{\alpha}(\mathcal{N}(t))
$$

has a solution in the form

$$
\begin{aligned}
\mathcal{N}(t)= & \mathcal{N}_{0} \sum_{\kappa=0}^{\infty} \frac{(-1)^{\kappa} \Gamma(n+2 \kappa+1)}{\kappa ! \Gamma(n+\kappa+1)}\left(\frac{t^{\alpha}}{2}\right)^{n+2 \kappa} \\
& \cdot E_{(1, n+2 \kappa+1)}\left(\frac{-d^{\alpha} t^{\alpha}}{\alpha}\right) .
\end{aligned}
$$

Proof. Following Abdeljawad [16], then, in virtue of $I_{\alpha}(f(t))$, we have

$$
L_{\alpha}\left\{I_{\alpha}(f(t))\right\}=\frac{F_{\alpha}(s)}{s},
$$

where $F_{\alpha}(s)=L_{\alpha}\{f(t)\}$ defined in (10) and $I_{\alpha}$ is the conformable fractional integral operator (2). Acting by the conformable fractional Laplace transform on both sides of Equation (30) implies that

$$
L_{\alpha}\{\mathcal{N}(t)\}-\mathcal{N}_{0} L_{\alpha}\left\{J_{n}^{\alpha}(t)\right\}=-d^{\alpha} L_{\alpha}\left\{I_{\alpha}(\mathcal{N}(t))\right\}
$$

Combining Equation (5) and Equation (31) leads to

$$
\begin{gathered}
\mathcal{N}_{\alpha}(s)-\mathcal{N}_{0} \sum_{\kappa=0}^{\infty} \frac{(-1)^{\kappa}}{\kappa ! \Gamma(n+\kappa+1) 2^{n+2 \kappa}} L_{\alpha}\left\{t^{\alpha(n+2 \kappa)}\right\} \\
=-d^{\alpha} \frac{\mathcal{N}_{\alpha}(s)}{s}
\end{gathered}
$$




$$
\begin{aligned}
\mathcal{N}_{\alpha}(s)\left(1+\frac{d^{\alpha}}{s}\right)= & \mathcal{N}_{0} \sum_{\kappa=0}^{\infty} \frac{(-1)^{\kappa}}{\kappa ! \Gamma(n+\kappa+1) 2^{n+2 \kappa}} \\
& \cdot \int_{0}^{\infty} e^{-s\left(t^{\alpha} / \alpha\right)} t^{\alpha(n+2 \kappa)} d_{\alpha} t .
\end{aligned}
$$

Considering Equation (10) with (34), we obtain

$\mathcal{N}_{\alpha}(s)\left(1+\frac{d^{\alpha}}{s}\right)=\mathcal{N}_{0} \sum_{\kappa=0}^{\infty} \frac{(-1)^{\kappa} \alpha^{(n+2 \kappa)}}{\kappa ! \Gamma(n+\kappa+1) 2^{n+2 \kappa}} \frac{\Gamma(n+2 \kappa+1)}{s^{n+2 \kappa+1}}$

Therefore,

$$
\begin{aligned}
\mathscr{N}_{\alpha}(s)= & \mathcal{N}_{0} \sum_{\kappa=0}^{\infty} \frac{(-1)^{\kappa} \alpha^{(n+2 \kappa)} \Gamma(n+2 \kappa+1)}{\kappa ! \Gamma(n+\kappa+1) 2^{n+2 \kappa}} \frac{1}{s^{n+2 \kappa+1}}\left(1+\frac{d^{\alpha}}{s}\right)^{-1} \\
= & \mathcal{N}_{0} \sum_{\kappa=0}^{\infty} \frac{(-1)^{k} \alpha^{(n+2 \kappa)} \Gamma(n+2 \kappa+1)}{\kappa ! \Gamma(n+\kappa+1) 2^{n+2 \kappa}} \\
& \cdot \frac{1}{s^{n+2 \kappa+1}} \sum_{i=0}^{\infty} \frac{(1)_{i}\left(-d^{\alpha} / s\right)^{i}}{i !} \\
= & \mathcal{N}_{0} \sum_{\kappa=0}^{\infty} \frac{(-1)^{\kappa} \alpha^{(n+2 k)} \Gamma(n+2 \kappa+1)}{\kappa ! \Gamma(n+\kappa+1) 2^{n+2 \kappa}} \sum_{i=0}^{\infty} \frac{(-1)^{i} d^{\alpha i}}{s^{n+2 \kappa+i+1}} .
\end{aligned}
$$

With the aid of the inverse Laplace transform (10), it follows that

$$
\begin{aligned}
\mathcal{N}(t)= & \mathcal{N}_{0} \sum_{\kappa=0}^{\infty} \frac{(-1)^{\kappa} \alpha^{(n+2 \kappa)} \Gamma(n+2 \kappa+1)}{\kappa ! \Gamma(n+\kappa+1) 2^{n+2 \kappa}} \\
& \cdot \sum_{i=0}^{\infty} \frac{(-1)^{i} d^{\alpha i} t^{\alpha(n+2 \kappa+i)}}{\alpha^{n+2 \kappa+i} \Gamma(n+2 \kappa+i+1)} \\
= & \mathcal{N}_{0} \sum_{\kappa=0}^{\infty} \frac{(-1)^{\kappa} \Gamma(n+2 \kappa+1)}{k ! \Gamma(n+\kappa+1)}\left(\frac{t^{\alpha}}{2}\right)^{n+2 \kappa} \\
& \cdot \sum_{i=0}^{\infty} \frac{1}{\Gamma(n+2 \kappa+i+1)}\left(\frac{-d^{\alpha} t^{\alpha}}{\alpha}\right)^{i} \\
= & \mathcal{N}_{0} \sum_{\kappa=0}^{\infty} \frac{(-1)^{\kappa} \Gamma(n+2 \kappa+1)}{\kappa ! \Gamma(n+\kappa+1)}\left(\frac{t^{\alpha}}{2}\right)^{n+2 \kappa} \\
& \cdot E_{(1, n+2 \kappa+1)}\left(\frac{-d^{\alpha} t^{\alpha}}{\alpha}\right) .
\end{aligned}
$$

Theorem 14. The solution of the following conformable fractional equation

$\mathcal{N}(t)-\mathcal{N}_{0}\left\{J_{n}^{\alpha}\left(d^{\mu} t^{\mu}\right)\right\}=-d^{\alpha \mu} I_{\alpha}(\mathcal{N}(t)), \quad$ for $d, \mu>0, \alpha \in(0,1]$, is given by

$$
\begin{aligned}
\mathcal{N}(t)= & \mathcal{N}_{0} \sum_{\kappa=0}^{\infty} \frac{(-1)^{\kappa} \Gamma(n \mu+2 \mu \kappa+1)}{\kappa ! \Gamma(n+\kappa+1)}\left(\frac{d^{\alpha \mu} t^{\alpha \mu}}{2}\right)^{n+2 \kappa} \\
& \cdot E_{(1, n \mu+2 \mu \kappa+1)}\left(\frac{-d^{\alpha} \mu t^{\alpha}}{\alpha}\right) .
\end{aligned}
$$

Proof. Operating the conformable fractional Laplace transform on both sides of Equation (38), we get

$$
L_{\alpha}\{\mathcal{N}(t)\}-\mathcal{N}_{0} L_{\alpha}\left\{J_{n}^{\alpha}\left(d^{\mu} t^{\mu}\right)\right\}=-d^{\alpha \mu} L_{\alpha}\left\{I_{\alpha}((t))\right\}
$$

In view of Equations (5) and (31), we obtain

$$
\begin{aligned}
\mathscr{N}_{\alpha}(s) & +d^{\alpha \mu} \frac{\mathcal{N}_{\alpha}(s)}{s} \\
& =N_{0} \sum_{\kappa=0}^{\infty} \frac{(-1)^{\kappa} d^{\mu \alpha(n+2 \kappa)}}{\kappa ! \Gamma(n+\kappa+1) 2^{n+2 \kappa}} L_{\alpha}\left\{t^{\alpha \mu(n+2 \kappa)}\right\} .
\end{aligned}
$$

Hence, using (3) of Lemma 7 implies

$$
\begin{aligned}
\mathcal{N}_{\alpha}(s)\left(1+\frac{d^{\alpha \mu}}{s}\right)= & \mathcal{N}_{0} \sum_{\kappa=0}^{\infty} \frac{(-1)^{\kappa} d^{\mu \alpha(n+2 \kappa)}}{\kappa ! \Gamma(n+\kappa+1) 2^{n+2 \kappa}} \\
& \cdot \frac{\alpha^{\mu(n+2 \kappa)} \Gamma(n \mu+2 \mu \kappa+1)}{s^{n \mu+2 \mu \kappa+1}},
\end{aligned}
$$

from which one can obtain

$$
\begin{aligned}
\mathcal{N}_{\alpha}(s)= & \mathcal{N}_{0} \sum_{\kappa=0}^{\infty} \frac{(-1)^{\kappa} d^{\mu \alpha(n+2 \kappa)} \alpha^{\mu(n+2 \kappa)} \Gamma(n \mu+2 \mu \kappa+1)}{\kappa ! \Gamma(n+\kappa+1) 2^{n+2 \kappa}} \\
& \cdot \frac{1}{s^{n \mu+2 \mu \kappa+1}} \sum_{i=0}^{\infty} \frac{(-1)^{i} d^{\alpha i} \mu^{i}}{s^{i}}
\end{aligned}
$$

Therefore,

$$
\begin{aligned}
\mathcal{N}_{\alpha}(s)= & \mathcal{N}_{0} \sum_{\kappa=0}^{\infty} \frac{(-1)^{\kappa} d^{\mu \alpha(n+2 \kappa)} \alpha^{\mu(n+2 \kappa)} \Gamma(n \mu+2 \mu \kappa+1)}{\kappa ! \Gamma(n+\kappa+1) 2^{n+2 \kappa}} \\
& \cdot \sum_{i=0}^{\infty}(-1)^{i} d^{\alpha i} \mu^{i} \frac{1}{s^{n \mu+2 \mu \kappa+i+1}} .
\end{aligned}
$$

In view of the inverse fractional Laplace transform (10), it follows that 


$$
\begin{aligned}
\mathcal{N}(t)= & \mathcal{N}_{0} \sum_{\kappa=0}^{\infty} \frac{(-1)^{\kappa} d^{\mu \alpha(n+2 \kappa)} \Gamma(n \mu+2 \mu \kappa+1)}{\kappa ! \Gamma(n+\kappa+1) 2^{n+2 \kappa}} t^{\alpha(n \mu+2 \mu \kappa)} \\
& \cdot \sum_{i=0}^{\infty} \frac{1}{\Gamma(n \mu+2 \mu \kappa+i+1)}\left[\frac{-d^{\alpha} \mu t^{\alpha}}{\alpha}\right]^{i} \\
= & \mathcal{N}_{0} \sum_{\kappa=0}^{\infty} \frac{(-1)^{\kappa} \Gamma(n \mu+2 \mu \kappa+1)}{\kappa ! \Gamma(n+\kappa+1)}\left[\frac{d^{\mu \alpha} t^{\alpha \mu}}{2}\right]^{n+2 \kappa} \\
& \cdot E_{(1, \mu n+2 \mu \kappa+1)}\left(\frac{-d^{\alpha} \mu t^{\alpha}}{\alpha}\right) .
\end{aligned}
$$

Thus, the result is established.

\section{Orthogonality of the CFBFs}

Understanding the orthogonality relation of the CFBFs is mandatory to compute coefficients of series whose terms include the CFBFs. These series represent solutions of the FDEs as we will encounter in the application part of this section. Along with [40] and in view of the CFD definition (1), we introduce the following interesting results on orthogonality which will be useful in the current study.

\subsection{An Orthogonal Relation of the CFBFs}

Theorem 15. The orthogonality relation of the CFBFs $J_{n}^{\alpha}(x)$ is deduced over $[0, b]$ with respect to the weight function $w(x)=x^{2 \alpha-1}$ by the following:

$$
\int_{0}^{b} x^{2 \alpha-1} J_{n}^{\alpha}\left(\lambda_{s} x\right) J_{n}^{\alpha}\left(\lambda_{r} x\right) d x=\frac{b^{2 \alpha}}{2 \alpha}\left[J_{n+1}^{\alpha}\left(\lambda_{s} b\right)\right]^{2} \delta_{\lambda_{s} \lambda_{r}}, \quad \alpha \in(0,1],
$$

where $\delta_{\lambda_{s} \lambda_{r}}$ is the familiar Kronker delta function and $\lambda_{s}, \lambda_{r}$ are distinct roots of $J_{n}^{\alpha}(x)=0$.

Proof. Since $J_{n}^{\alpha}(x)$ is a solution of the CFBE (4), it follows that $y=J_{n}^{\alpha}\left(\lambda_{s} x\right)$ which satisfies the more general equation

$$
x^{2 \alpha} \mathscr{D}^{\alpha} \mathscr{D}^{\alpha} y(x)+\alpha x^{\alpha} \mathscr{D}^{\alpha} y(x)+\alpha^{2}\left(x^{2 \alpha} \lambda_{s}^{2 \alpha}-n^{2}\right) y(x)=0 .
$$

It is convenient to reformulate (47) in the following way:

$$
x^{\alpha} \mathscr{D}^{\alpha}\left(x^{\alpha} \mathscr{D}^{\alpha} y(x)\right)+\alpha^{2}\left(x^{2 \alpha} \lambda_{s}^{2 \alpha}-n^{2}\right) y(x)=0 .
$$

Consequently, $J_{n}^{\alpha}\left(\lambda_{s} x\right)$ and $J_{n}^{\alpha}\left(\lambda_{r} x\right)$ satisfy the following CFDEs, respectively:

$$
\begin{aligned}
& x^{\alpha} \mathscr{D}^{\alpha}\left(x^{\alpha} \mathscr{D}^{\alpha} J_{n}^{\alpha}\left(\lambda_{s} x\right)\right)+\alpha^{2}\left(x^{2 \alpha} \lambda_{s}^{2 \alpha}-n^{2}\right) J_{n}^{\alpha}\left(\lambda_{s} x\right)=0, \\
& x^{\alpha} \mathscr{D}^{\alpha}\left(x^{\alpha} \mathscr{D}^{\alpha} J_{n}^{\alpha}\left(\lambda_{r} x\right)\right)+\alpha^{2}\left(x^{2 \alpha} \lambda_{r}^{2 \alpha}-n^{2}\right) J_{n}^{\alpha}\left(\lambda_{r} x\right)=0 .
\end{aligned}
$$

Multiplying (49) by $x^{-\alpha} J_{n}^{\alpha}\left(\lambda_{r} x\right)$ and (50) by $x^{-\alpha} J_{n}^{\alpha}\left(\lambda_{s} x\right)$ and then subtracting the resulting equations produce

$$
\begin{aligned}
& \left(\lambda_{s}^{2 \alpha}-\lambda_{r}^{2 \alpha}\right) x^{\alpha} J_{n}^{\alpha}\left(\lambda_{s} x\right) J_{n}^{\alpha}\left(\lambda_{r} x\right) \\
& \quad=J_{n}^{\alpha}\left(\lambda_{s} x\right) \mathscr{D}^{\alpha}\left[x^{\alpha} \mathscr{D}^{\alpha} J_{n}^{\alpha}\left(\lambda_{r} x\right)\right]-J_{n}^{\alpha}\left(\lambda_{r} x\right) \mathscr{D}^{\alpha}\left[x^{\alpha} \mathscr{D}^{\alpha} J_{n}^{\alpha}\left(\lambda_{s} x\right)\right] .
\end{aligned}
$$

In view of the conformable fractional integral formula (2) over $[0, b]$, we obtain

$$
\begin{gathered}
\left(\lambda_{s}^{2 \alpha}-\lambda_{r}^{2 \alpha}\right) \int_{0}^{b} x^{\alpha} J_{n}^{\alpha}\left(\lambda_{s} x\right) J_{n}^{\alpha}\left(\lambda_{r} x\right) d_{\alpha} x \\
=\int_{0}^{b} J_{n}^{\alpha}\left(\lambda_{s} x\right) \mathscr{D}^{\alpha}\left[x^{\alpha} \mathscr{D}^{\alpha} J_{n}^{\alpha}\left(\lambda_{r} x\right)\right] d_{\alpha} x \\
\quad-\int_{0}^{b} J_{n}^{\alpha}\left(\lambda_{r} x\right) \mathscr{D}^{\alpha}\left[x^{\alpha} \mathscr{D}^{\alpha} J_{n}^{\alpha}\left(\lambda_{s} x\right)\right] d_{\alpha} x .
\end{gathered}
$$

By performing integration by parts [16] on the righthand side divided by the factor $\left(\lambda_{s}^{2 \alpha}-\lambda_{r}^{2 \alpha}\right)$, one can conclude that

$$
\begin{aligned}
\int_{0}^{b} x^{\alpha} J_{n}^{\alpha}\left(\lambda_{s} x\right) J_{n}^{\alpha}\left(\lambda_{r} x\right) d_{\alpha} x= & \frac{x^{\alpha}}{\left(\lambda_{s}^{2 \alpha}-\lambda_{r}^{2 \alpha}\right)}\left[J_{n}^{\alpha}\left(\lambda_{s} x\right) \mathscr{D}^{\alpha} J_{n}^{\alpha}\left(\lambda_{r} x\right)\right. \\
& \left.-J_{n}^{\alpha}\left(\lambda_{r} x\right) \mathscr{D}^{\alpha} J_{n}^{\alpha}\left(\lambda_{s} x\right)\right]_{0}^{b}
\end{aligned}
$$

Hence, according to the values of $\lambda_{s}$ and $\lambda_{r}$, we consider the following two cases:

(i) If $\lambda_{s} \neq \lambda_{r}$ and by hypothesis $J_{n}^{\alpha}\left(\lambda_{s}\right)=J_{n}^{\alpha}\left(\lambda_{r}\right)=0$, then the right-hand side of (53) vanishes

(ii) If $\lambda_{s}=\lambda_{r}$, then the resulting integral

$$
I=\int_{0}^{b} x^{\alpha}\left[J_{n}^{\alpha}\left(\lambda_{s} x\right)\right]^{2} d_{\alpha} x=\int_{0}^{b} x^{2 \alpha-1}\left[J_{n}^{\alpha}\left(\lambda_{s} x\right)\right]^{2} d x
$$

creates an interest to look at. In order to deduce its value, we take the limit of (53) as $\lambda_{r} \longrightarrow \lambda_{s}$. As the right-hand side in (53) approaches the indeterminate form $0 / 0$ in the limit, we apply L'Hopital's rule, which leads to

$$
I=\frac{x^{\alpha}}{2 \alpha \lambda_{s}^{\alpha}}\left[\mathscr{D}_{\lambda_{s}}^{\alpha} J_{n}^{\alpha}\left(\lambda_{s} x\right) \mathscr{D}_{x}^{\alpha} J_{n}^{\alpha}\left(\lambda_{s} x\right)-J_{n}^{\alpha}\left(\lambda_{s} x\right) \mathscr{D}_{\lambda_{s}}^{\alpha} \mathscr{D}_{x}^{\alpha} J_{n}^{\alpha}\left(\lambda_{s} x\right)\right]_{0}^{b}
$$
[26]

Now, using the following recurrence relations of CFBFs

$$
\begin{aligned}
& \mathscr{D}_{\lambda_{s}}^{\alpha} J_{n}^{\alpha}\left(\lambda_{s} x\right)=\frac{n}{x^{\alpha}} J_{n}^{\alpha}\left(\lambda_{s} x\right)-\lambda_{s}^{\alpha} J_{n+1}^{\alpha}\left(\lambda_{s} x\right), \\
& \mathscr{D}_{x}^{\alpha} J_{n}^{\alpha}\left(\lambda_{s} x\right)=\frac{n}{\lambda_{s}^{\alpha}} J_{n}^{\alpha}\left(\lambda_{s} x\right)-x^{\alpha} J_{n+1}^{\alpha}\left(\lambda_{s} x\right),
\end{aligned}
$$




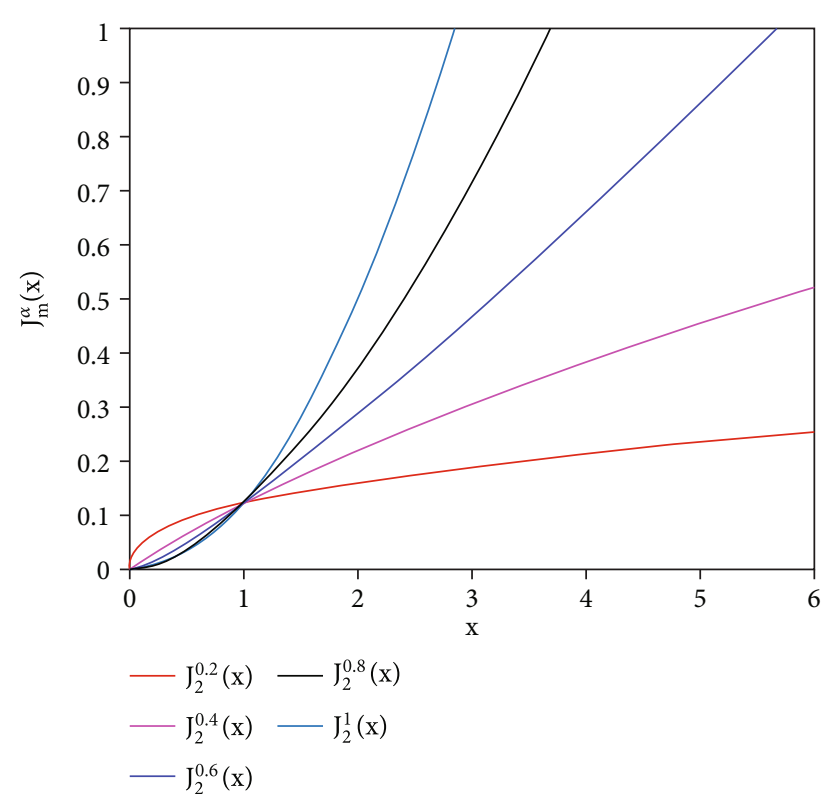

Figure 1: Graph of CFBFs with $\mathscr{M}=2, m=2$ and various values of $\alpha=0.2,0.4,0.6,0.8,1$.

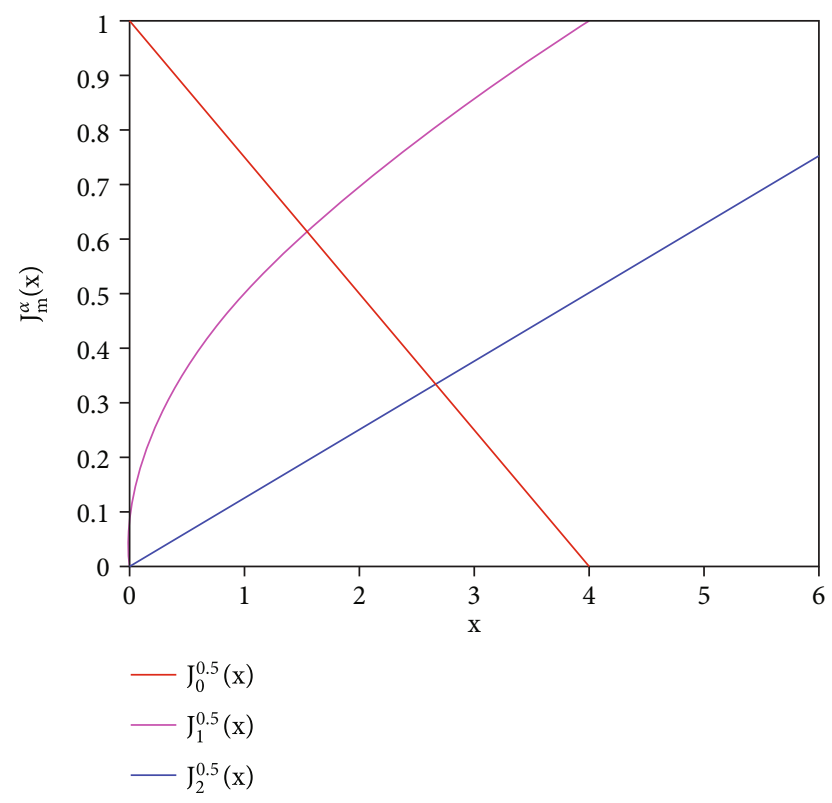

FIgURE 2: Graph of CFBFs with $\mathscr{M}=2, \alpha=0.5$ and various values of $m=0,1,2$.

it follows that

$$
\begin{aligned}
I= & {\left[\frac{n^{2}}{2 \alpha \lambda_{s}^{2 \alpha}}\left[J_{n}^{\alpha}\left(\lambda_{s} x\right)\right]^{2}+\frac{x^{2 \alpha}}{2 \alpha}\left[J_{n+1}^{\alpha}\left(\lambda_{s} x\right)\right]^{2}\right.} \\
& \left.-\frac{n x^{\alpha}}{2 \alpha \lambda_{s}^{\alpha}} J_{n}^{\alpha}\left(\lambda_{s} x\right) J_{n+1}^{\alpha}\left(\lambda_{s} x\right)\right]_{0}^{b}=\frac{b^{2 \alpha}}{2 \alpha}\left[J_{n+1}^{\alpha}\left(\lambda_{s} b\right)\right]^{2} .
\end{aligned}
$$

As a special case of Theorem 15, the following result can be easily verified.
Corollary 16. The CFBFs $J_{n}^{\alpha}(x)$ are orthogonal over $[0,1]$ with respect to the weight function $w(x)=x^{2 \alpha-1}$ and

$$
\int_{0}^{1} x^{2 \alpha-1} J_{n}^{\alpha}\left(\lambda_{s} x\right) J_{n}^{\alpha}\left(\lambda_{r} x\right) d x=\frac{1}{2 \alpha}\left[J_{n+1}^{\alpha}\left(\lambda_{s}\right)\right]^{2} \delta_{\lambda_{s} \lambda_{r}} .
$$

The results obtained in the current Section 4.1 treated the topic of orthogonal polynomials which pave the way to discuss a function representation via a series of the CFBFs.

4.2. Expansion of Functions via CFBFs. The classical theory of expressing analytic functions as expansions in terms of an arbitrary set of orthogonal polynomials can be described as the backbone of many topics in analysis. It was originated by several authors to whom we may mention Boas and Buck [41], Faber [42], and Whittaker and Gattegno [43] and later on in higher dimension by Abul-Ez et al. [29, 30, 44-46]. In the usual classical calculus, we found that not all functions have the Taylor power series representation around specific points, but this is not the case in the theory of conformable fractional calculus. This fact has been shown by Abdeljawad [16], where he also proposed the expansion of the fractional power series for an infinity $\alpha$-differentiable function through the fractional Taylor series. The expansion of a given real function in a series of Bessel functions is extremely useful in determining the solution of certain FDEs involving radial symmetry [40]. Related to the work of finding the expansion of a given function by means of Bessel polynomials in the higher-dimensional context, see the work given in $[29,30]$. Using the orthogonality property (46), one can easily represent a given function $f(x)$ over the interval $[0, b]$ by a series of Bessel functions such as

$$
f(x)=\sum_{i=0}^{\infty} a_{i} J_{n}^{\alpha}\left(\lambda_{i} x\right), \quad 0<x<b,
$$

where $J_{n}^{\alpha}\left(\lambda_{i} b\right)=0, i=0,1,2,3, \cdots$, and $a_{i}$ are determined by

$$
a_{i}=\frac{2 \alpha}{b^{2 \alpha}\left[J_{n+1}^{\alpha}\left(\lambda_{i} b\right)\right]^{2}} \int_{0}^{b} x^{2 \alpha-1} f(x) J_{n}^{\alpha}\left(\lambda_{i} x\right) d x, \quad i=0,1,2,3, \cdots
$$

As the topic of expansions of an arbitrary function, of either a real or a complex variable, into a series of polynomials has not been fully explored, we believe that several open problems remain untouched in particular in the framework of fractional calculus.

4.3. Applications. In this subsection, we intend to indicate the efficiency and applicability of the results developed in this study. Precisely, we construct a scheme which will be employed to solve some linear and nonlinear CFDEs. In this concern, we first define the $m^{\text {th }}$ truncated CFBFs of the first kind. Then, we introduce the noninteger derivative in the conformable context of an approximated function expanded in terms of the CFBFs. Several important examples including famous FDEs have been comprehensively treated, and their solutions have been compared to other existing methods in the literature to show the consistency and accuracy of our proposed method. 
We begin by considering the $m^{\text {th }}$ truncated CFBFs of the first kind as follows:

$$
\tilde{J}_{m}^{\alpha}(x)=\sum_{\kappa=0}^{\lfloor(\mathcal{M}-m) / 2\rfloor} \frac{(-1)^{\kappa}}{\kappa ! \Gamma(m+\kappa+1)}\left(\frac{x^{\alpha}}{2}\right)^{2 \kappa+m}, \quad 0 \leq x<\infty,
$$

where $\mathscr{M}$ is a positive integer such that $\mathscr{M} \geq m$ and $m=0$, $1,2, \cdots, \mathscr{M}$. For $\mathscr{M}=2$, we have

$$
\begin{aligned}
& \tilde{J}_{0}^{\alpha}(x)=1-\frac{x^{2 \alpha}}{4}, \\
& \tilde{J}_{1}^{\alpha}(x)=\frac{x^{\alpha}}{2} \\
& \tilde{J}_{m}^{2}(x)=\frac{x^{2 \alpha}}{8} .
\end{aligned}
$$

Figures 1 and 2 show the graphs of the truncated CFBFs when $\mathscr{M}=2$ and by taking various values of $m$ and $\alpha$.

Let $f(x)$ be a function defined over $[0,1]$; then, $f(x)$ can be expanded in terms of CFBFs as follows:

$$
f(x)=\sum_{i=0}^{\infty} a_{i} \tilde{J}_{i}^{\alpha}(x) .
$$

Thus, the following truncated series for $f(x)$ is supposed to be

$$
f(x)=\sum_{m=0}^{\mathscr{M}} a_{m} \tilde{J}_{m}^{\alpha}(x)
$$

Theorem 17. The noninteger derivative of order $\gamma>0$ of the CFBFs in the conformable sense is given by

$$
\mathscr{D}^{\gamma} \tilde{J}_{m}^{\alpha}(x)=\sum_{\kappa=0}^{\lfloor(\mathscr{M}-m) / 2\rfloor} \eta_{\kappa, m}^{\alpha, \gamma} x^{\alpha(2 \kappa+m)-\gamma},
$$

where

$$
\eta_{\kappa, \kappa}^{\alpha, \gamma}=\frac{(-1)^{\kappa} \Gamma(\alpha(2 \kappa+m)+1)}{2^{2 \kappa+m} k ! \Gamma(m+\kappa+1) \Gamma(\alpha(2 \kappa+m)-\lceil\gamma\rceil+1)} .
$$

Proof. The linearity of the conformable derivative (see [12, 16]) leads to

$$
\begin{aligned}
\mathscr{D}^{\gamma} \tilde{J}_{m}^{\alpha}(x)= & \sum_{\kappa=0}^{\lfloor(\mathcal{M}-m) / 2\rfloor} \frac{(-1)^{\kappa}}{2^{2 \kappa+m} \kappa ! \Gamma(m+\kappa+1)} \mathscr{D}^{\gamma} x^{\alpha(2 \kappa+m)} \\
= & \sum_{\kappa=0}^{\lfloor(\mathcal{M}-m) / 2\rfloor} \frac{(-1)^{\kappa}}{2^{2 \kappa+m} \kappa ! \Gamma(m+\kappa+1)} \\
& \cdot \frac{\Gamma(\alpha(2 \kappa+m)+1)}{\Gamma(\alpha(2 \kappa+m)-\lceil\gamma\rceil+1)} x^{\alpha(2 \kappa+m)-\gamma},
\end{aligned}
$$

as required.
Remark 18. If $\alpha(2 \kappa+m)<\gamma$, where $\alpha(2 \kappa+m) \in \mathbb{N}_{0}$, then, $\mathscr{D}^{\gamma} \tilde{J}_{m}^{\alpha}(x)=0$.

Theorem 19. Let $u_{M}(x)$ be an approximated function given by means of the truncated formula of CFBFs (61). Then,

$$
\mathscr{D}^{\gamma} u_{\mathscr{M}}(x)=\sum_{m=0}^{\mathscr{M}} \sum_{\kappa=0}^{\lfloor(\mathcal{M}-m) / 2\rfloor} a_{m} \eta_{\kappa, m}^{\alpha, \gamma} x^{\alpha(2 \kappa+m)-\gamma} .
$$

Proof. The induction of Theorem 17 and in view of the linearity property leads to the required result.

\subsubsection{Proposed Scheme}

(1) Linear Multiorder CFDEs. Suppose that the generalized linear multiorder CFDE is given in the form

$$
\mathscr{D}^{\gamma} u(x)+\sum_{j=0}^{s} A_{j} \mathscr{D}^{\gamma_{j}} u(x)+A_{s+1} u(x)=A_{s+2} h(x), \in[0,1],
$$

subject to the initial conditions

$$
\mathscr{D}^{(i)} u(x)=d_{i}, i=0,1,2, \cdots,\lceil\gamma\rceil-1,
$$

where $\mathscr{D}^{\gamma}, 0<\gamma_{1}<\gamma_{2}<\cdots<\gamma_{s}<\gamma$, denotes to the CFD of order $\gamma>0, h(x)$ are known to be a continuous function, and $d_{i}, i=0,1,2, \cdots,\lceil\gamma\rceil-1$ are some constants.

Assume that the solution of the CFDE (69) can be given in the form

$$
u_{M}(x)=\sum_{m=0}^{\mathscr{M}} a_{m} \tilde{J}_{m}^{\alpha}(x)
$$

Substituting (71) into (69) and using Theorem 19, we have

$$
\begin{aligned}
& \sum_{m=0}^{\mathscr{M}} \sum_{\kappa=0}^{\lfloor(\mathcal{M}-m) / 2\rfloor} a_{m} \eta_{\kappa, m}^{\alpha, \gamma} x^{\alpha(2 \kappa+m)-\gamma} \\
& +\sum_{j=0}^{s} A_{j}\left\{\sum_{m=0}^{\mathscr{M}} \sum_{\kappa=0}^{\lfloor(\mathscr{M}-m) / 2\rfloor} a_{m} \eta_{\kappa, m}^{\alpha, \gamma_{j}} x^{\alpha(2 \kappa+m)-\gamma_{j}}\right\} \\
& +A_{s+1} \sum_{m=0}^{\mathscr{M}} a_{m} \tilde{J}_{m}^{\alpha}(x)=A_{s+2} h(x) .
\end{aligned}
$$

Collocating Equation (72) at the points $x_{q}=q / \mathscr{M},=1$, $2,3, \cdots, \mathscr{M}+1-\lceil\gamma\rceil$, we get 


$$
\begin{aligned}
& \sum_{m=0}^{\mathscr{M}} \sum_{\kappa=0}^{\lfloor(\mathscr{M}-m) / 2\rfloor} a_{m} \eta_{\kappa, m}^{\alpha, \gamma} x_{q}^{\alpha(2 \kappa+m)-\gamma} \\
& \quad+\sum_{j=0}^{s} A_{j}\left\{\sum_{m=0}^{\mathscr{M}} \sum_{\kappa=0}^{\lfloor(\mathscr{M}-m) / 2\rfloor} a_{m} \eta_{\kappa, m}^{\alpha, \gamma_{j}} x_{q}^{\alpha(2 \kappa+m)-\gamma_{j}}\right\} \\
& \quad+A_{s+1} \sum_{m=0}^{\mathscr{M}} a_{m} \tilde{J}_{m}^{\alpha}\left(x_{q}\right)=A_{s+2} h\left(x_{q}\right) .
\end{aligned}
$$

These equations give $(\mathscr{M}+1-\lceil\gamma\rceil)$ linear algebraic equations. Combining (71) and (70) produces $\lceil\gamma\rceil$-algebraic equations. Thus, we obtain a linear algebraic system of $\mathscr{M}$ +1 equations in the unknown $a_{m}, m=0,1,2, \cdots, \mathscr{M}$. By solving this system, we obtain the solution of Equation (69) associated with the initial condition (70).

(2) Nonlinear Multiorder CFDEs. Consider the generalized nonlinear multiorder CFDE such that

$$
\begin{aligned}
\mathscr{D}^{\gamma} u(x)= & F\left(x, u(x), \mathscr{D}^{\gamma_{1}} u(x), \mathscr{D}^{\gamma^{2}} u(x), \cdots,\right. \\
& \left.\mathscr{D}^{\gamma_{s}} u(x)\right), \quad x \in[0,1],
\end{aligned}
$$

with the initial conditions

$$
\mathscr{D}^{(i)} u(x)=c_{i}, i=0,1,2, \cdots,\lceil\gamma\rceil-1 \text {, }
$$

where $\mathscr{D}^{\gamma}, 0<\gamma_{1}<\gamma_{2}<\cdots<\gamma_{s}<\gamma$, stands for the CFD of order $\gamma>0, F$ is a nonlinear operator, and $c_{i}, i=0,1,2, \cdots$, $\lceil\gamma\rceil-1$ are given constants. A similar procedure as in establishing (69) can be used to approximate $u(x)$ in terms of CFBFs. Thus, combining (71) with (74) and using Theorem 19 yield

$$
\begin{aligned}
& \sum_{m=0}^{\mathscr{M}} \sum_{\kappa=0}^{\lfloor(\mathscr{M}-m) / 2\rfloor} a_{m} \eta_{\kappa, m}^{\alpha, \gamma} x^{\alpha(2 \kappa+m)-\gamma} \\
& =F\left(x, \sum_{m=0}^{\mathscr{M}} a_{m} \tilde{J}_{m}^{\alpha}(x), \sum_{m=0}^{\mathscr{M}} \sum_{\kappa=0}^{\lfloor(\mathscr{M}-m) / 2\rfloor}\right. \\
& \cdot a_{m} \eta_{\kappa, m}^{\alpha, \gamma_{1}} x^{\alpha(2 \kappa+m)-\gamma_{1}}, \sum_{m=0}^{M^{\lfloor(M-m) / 2\rfloor}} \sum_{\kappa=0}^{\lfloor(\mathscr{M}-m) / 2\rfloor} \\
& \cdot a_{m} \eta_{\kappa, m}^{\alpha, \gamma_{2}} x^{\alpha(2 \kappa+m)-\gamma_{2}}, \cdots, \sum_{m=0}^{\left\lfloor\sum_{\kappa=0}\right.} \\
& \left.\cdot a_{m} \eta_{\kappa, m}^{\alpha, \gamma_{s}} x^{\alpha(2 \kappa+m)-\gamma_{s}}\right) .
\end{aligned}
$$

Collocating Equation (76) at the points $x_{q},=1,2,3, \cdots$, $\mathscr{M}+1-\lceil\gamma\rceil$, thus, we have the following:

$$
\begin{aligned}
& \sum_{m=0}^{\mathscr{M}} \sum_{\kappa=0}^{\lfloor(\mathscr{M}-m) / 2\rfloor} a_{m} \eta_{\kappa, m}^{\alpha, \gamma} x_{q}^{\alpha(2 \kappa+m)-\gamma} \\
& =F\left(x_{q}, \sum_{m=0}^{\mathscr{M}} a_{m} \tilde{J}_{m}^{\alpha}\left(x_{q}\right), \sum_{m=0}^{\mathscr{M}} \sum_{\kappa=0}^{\lfloor(\mathscr{M}-m) / 2\rfloor}\right. \\
& \cdot a_{m} \eta_{\kappa, m}^{\alpha, \gamma_{1}} x_{q}^{\alpha(2 \kappa+m)-\gamma_{1}}, \sum_{m=0}^{\mathscr{M}} \sum_{\kappa=0}^{\lfloor(\mathscr{M}-m) / 2\rfloor} \\
& \cdot a_{m} \eta_{\kappa, m}^{\alpha, \gamma_{2}} x_{q}^{\alpha(2 \kappa+m)-\gamma_{2}}, \cdots, \sum_{m=0}^{\mathscr{M}} \sum_{\kappa=0}^{\lfloor(\mathscr{M}-m) / 2\rfloor} \\
& \left.\cdot a_{m} \eta_{\kappa, m}^{\alpha, \gamma_{s}} x_{q}^{\alpha(2 \kappa+m)-\gamma_{s}}\right)
\end{aligned}
$$

Equation (77) determines $(\mathscr{M}+1-\lceil\gamma\rceil)$ nonlinear equations. In virtue of (71) and the initial conditions (75), we get $\lceil\gamma\rceil$ equations. Immediately, one can get a system of $\mathscr{M}+1$ nonlinear equations in the unknown $a_{m}, m=0,1,2, \cdots, \mathscr{M}$. As usual, Newton's iterative method can be used to solve this system. Thus, the solution of the nonlinear CFDE (74) with (75) can be deduced.

We can briefly clarify the achieved advantages of employing the proposed method as follows. The fractionalorder Bessel functions approximate the fractional function with more accuracy. This feature has made the FBFs more effective than Bessel functions in solving the fractional problems. As the values of coefficients in Bessel polynomials are smaller than the coefficients of Chebyshev, Legendre, and Bernoulli polynomials, the computational error in the current method is less. Furthermore, our detailed treatment to these FDEs using the collocation method is aimed at encouraging the use of such approach which allows reaching the required solutions with ease and accuracy.

In the following subsection, we discuss several numerical examples to demonstrate the consequences of the abovementioned features.

4.4. Illustrative Examples. In view of the above arguments, it is interesting to employ the provided techniques to solve some useful FDEs, as we shall see through the following examples.

Example 20. Consider the famous Bagley-Torvik equation

$$
\mathscr{D}^{2} u(x)+\mathscr{D}^{3 / 2} u(x)+u(x)=1+x, \quad 0 \leq x \leq 1,
$$

subject to the initial conditions

$$
u(0)=1, u^{\prime}(0)=1
$$

This equation has been solved using Legendre polynomials in view of the conformable fractional sense in [14], as well as in [47-50] in the sense of Caputo derivative. The exact solution of $(78)$ was given in $([47,49])$ as $u(x)=1+$ 
TABLE 1

Conformable fractional differential equation

Initial conditions

Exact solution

Values of $\mathscr{M}$ and $\alpha$, as indicated in relation (71)

Coefficients to be determined in relation (71)

Solution

$$
\begin{gathered}
\mathscr{D}^{3} u(x)+\mathscr{D}^{5 / 2} u(x)+u^{2}(x)=x^{4}, x \in[0,1] \\
u(0)=0, u^{\prime}(0)=0, \text { and } u^{\prime \prime}(0)=2 \\
x^{2}(\text { as given by various authors in }[47-50]) \\
\mathscr{M}=4 \text { and } \alpha=1 \\
a_{i}=0 \text { for } i=0,1,3, a_{2}=8 \text {, and } a_{4}=32
\end{gathered}
$$

$x^{2}$

TABLE 2

Conformable fractional differential equation

Initial conditions

Exact solution

Values of $\mathscr{M}$ and $\alpha$, as is indicated in relation (71).

Coefficients to be determined in relation (71)

Solution

$$
\begin{gathered}
\mathscr{D}^{4} u(x)+\mathscr{D}^{7 / 2} u(x)+u^{3}(x)=x^{9}, 0<x \leq 1 \\
u(0)=0, u^{\prime}(0)=0, u^{\prime \prime}(0)=0 \text {, and } u^{\prime \prime \prime}(0)=6 \\
\left.x^{3} \text { (as given in }[51,52]\right) \\
\mathscr{M}=4 \text { and } \alpha=1 \\
a_{i}=0 \text { for } i=0,1,2,4, a_{3}=48
\end{gathered}
$$

$x^{3}$

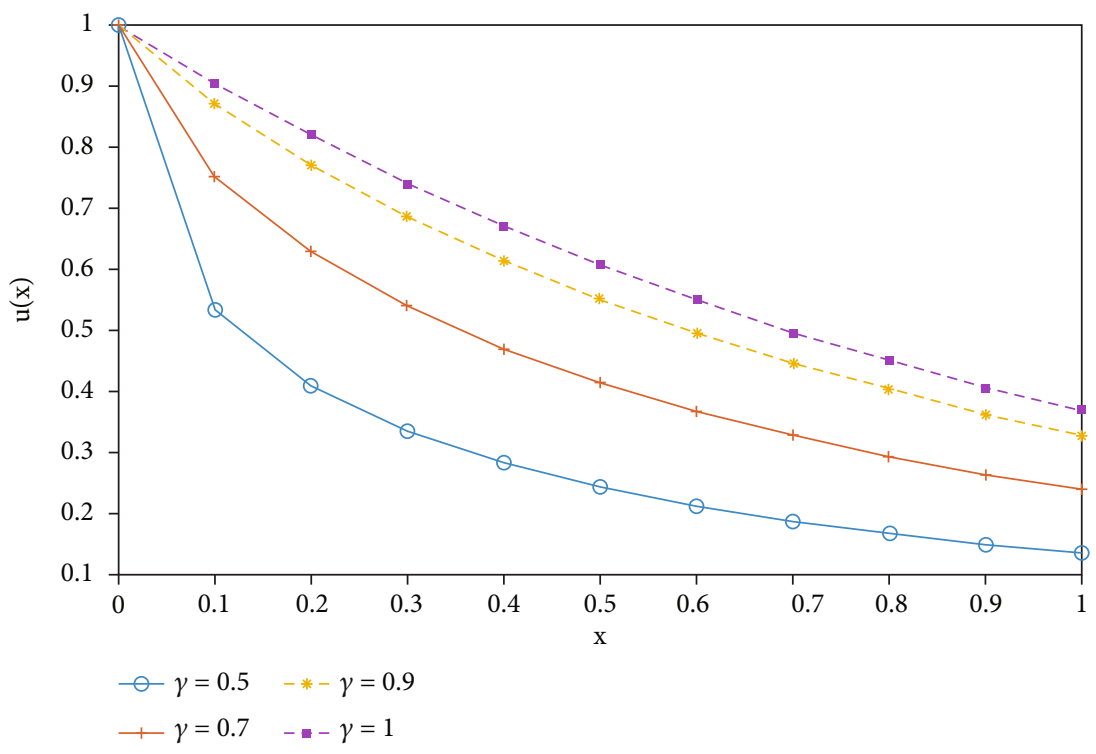

FIgURE 3: Graph of the approximate solutions of Example 22 when $\mathscr{M}=5$ with $\gamma=\alpha=0.5,0.7,0.9,1$.

$x$; then for $\mathscr{M}=2$ and $\alpha=1$ in (71), one gets the approximated solution of (78) in the form

$$
u_{2}(x)=\sum_{m=0}^{2} a_{m} \tilde{J}_{m}^{\alpha}(x)
$$

Owing to initial condition (79), then (80) gives

$$
a_{0}=1, a_{1}=2
$$

Using the collocation point $x=0.5$ and in view of (81), one can get

$$
0.3982 a_{2}=0.7964 \text {. }
$$

Hence, $a_{2}=2$. Therefore, we have $u(x)=1+x$, which coincides with the one given in $([47,49])$.

A similar procedure can be carried out as in Example 20, so that we may summarize the corresponding details for two different CFDEs as follows.

\section{Remark 21.}

(i) The problem in Table 1 has been treated in [47-50] with the Caputo fractional derivative using various methods such as Legendre polynomials, homotopy perturbation method, and homotopy analysis method

(ii) The problem in Table 2 has been manipulated in [51] by means of Legendre polynomials with conformable 

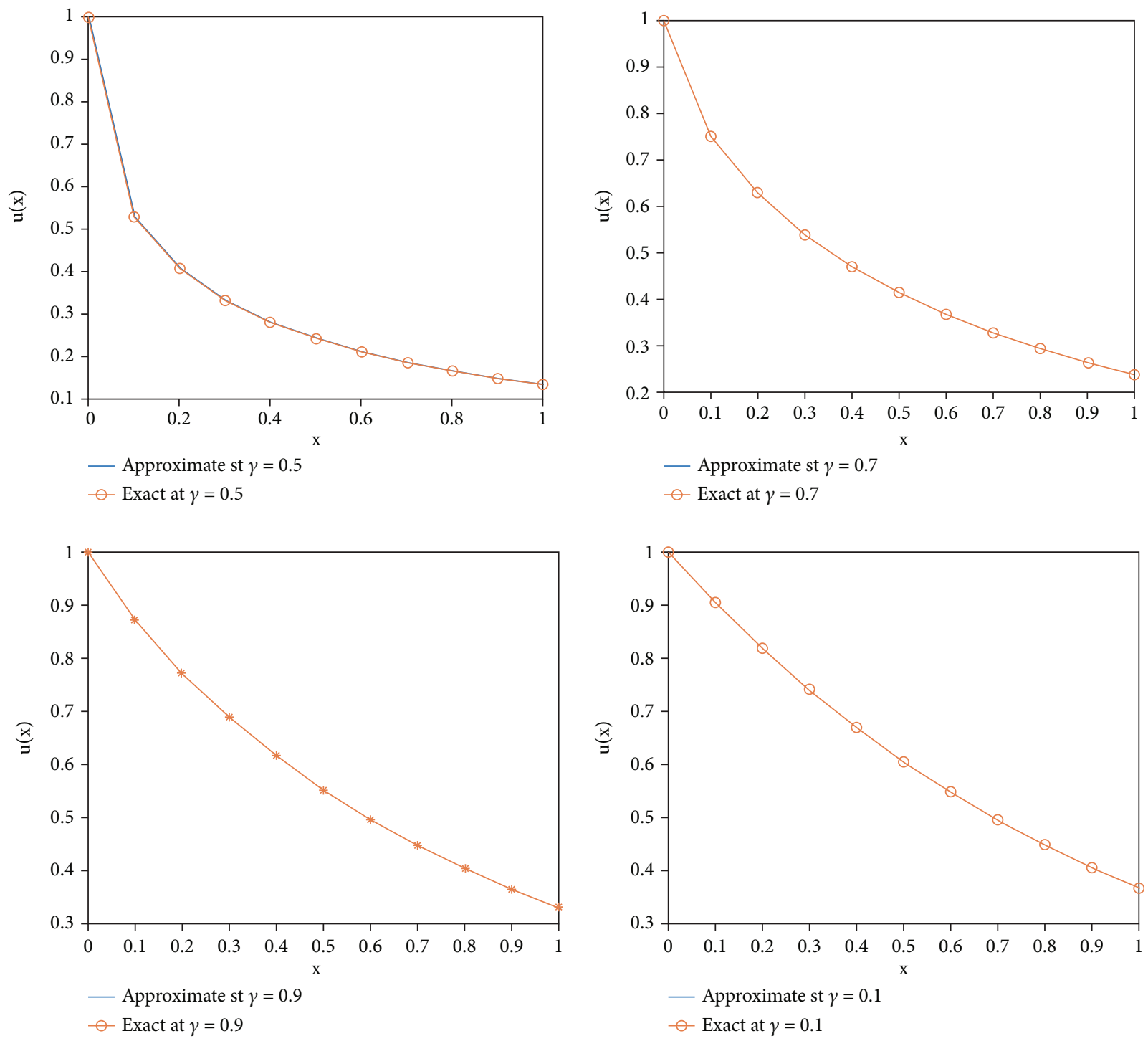

Figure 4: The comparison of the approximate solutions of Example 22 with the exact solution when $\mathscr{M}=5$ and for $\gamma=\alpha=0.5,0.7,0.9,1$.

derivative and in [52] in view of Laguerre polynomials involving Caputo derivative

Example 22. Suppose that we have the following linear fractional differential equation in the form

$$
\mathscr{D}^{\gamma} u(x)+u(x)=0, \quad 0<\gamma \leq 1,
$$

with the initial condition

$$
u(0)=1
$$

The exact solution of $(83)$ is $u(x)=\exp \left(-\left(x^{\gamma} / \gamma\right)\right)$ (see [47]). This problem has been evaluated using various methods (see for example $[47,49,53]$ ) in the sense of the Caputo fractional derivative. Putting $\mathscr{M}=5$ in (71) with $\gamma$ $=\alpha$, then using the presented technique, we computed the approximate solution of the problem (83) for the values $\gamma$ $=\alpha=0.5,0.7,0.9,1$. We indicate the approximate solution through Figure 3, while Figure 4 compares the obtained solution with the exact solution of (83). Moreover, the corresponding absolute errors of our approximate solution are displayed in Table 3. From Figure 4, it is clear that the approximate solutions converge to the exact solutions.

Example 23. It is well known that the theory of Bessel functions is connected with the Riccati equations. In fact, Bessel functions are defined as solutions of Bessel equations, which can be derived from the Riccati equations. This motivates us to consider the following the nonlinear Riccati fractional differential equation:

$$
\mathscr{D}^{\gamma} u(x)+u^{2}(x)=1, \quad 0<\gamma, x \leq 1
$$

subject to the initial condition

$$
u(0)=0
$$


TABLe 3: Absolute errors for example 22 in the case of $\mathscr{M}=5$ with various values of $\gamma=\alpha$.

\begin{tabular}{lcccc}
\hline$x$ & $\alpha=0.5$ & $\alpha=0.7$ & $\alpha=0.9$ & $\alpha=1$ \\
\hline 0.1 & $1.698913766905 .10^{-3}$ & $2.01482483935533 .10^{-4}$ & $2.55260108363852 .10^{-5}$ & $9.4292936385015 .10^{-6}$ \\
0.2 & $1.341809039425 .10^{-3}$ & $1.80206291971352 .10^{-4}$ & $2.57393080246859 .10^{-5}$ & $1.0100445353045 .10^{-5}$ \\
0.3 & $1.094073239144 .10^{-3}$ & $1.53118426981402 .10^{-4}$ & $2.22828517444373 .10^{-5}$ & $8.740914169325 .10^{-6}$ \\
0.4 & $9.222016584745 .10^{-4}$ & $1.32711916546735 .10^{-4}$ & $1.9580499485472 .10^{-5}$ & $7.674994977578 .10^{-6}$ \\
0.5 & $7.94771487391 .10^{-4}$ & $1.17204363458189 .10^{-4}$ & $1.77639190854228 .10^{-5}$ & $7.0847477455521 .10^{-6}$ \\
0.6 & $6.94723214864 .10^{-4}$ & $1.04225706794691 .10^{-4}$ & $1.61290436707651 .10^{-5}$ & $6.5282543029262 .10^{-6}$ \\
0.7 & $6.13410718572 .10^{-4}$ & $9.28101826830340 .10^{-5}$ & $1.43823651976849 .10^{-5}$ & $5.7858773236572 .10^{-6}$ \\
0.8 & $5.46280728516 .10^{-4}$ & $8.30201804029595 .10^{-5}$ & $1.2815490789575 .10^{-5}$ & $5.0984705504265 .10^{-6}$ \\
0.9 & $4.90369742377 .10^{-4}$ & $7.50420455738476 .10^{-5}$ & $1.18437600535870 .10^{-5}$ & $4.8554037531864 .10^{-6}$ \\
1 & $4.42903116775 .10^{-4}$ & $6.82330917218671 .10^{-5}$ & $1.11289526305072 .10^{-5}$ & $4.7847594708325 .10^{-6}$ \\
\hline
\end{tabular}

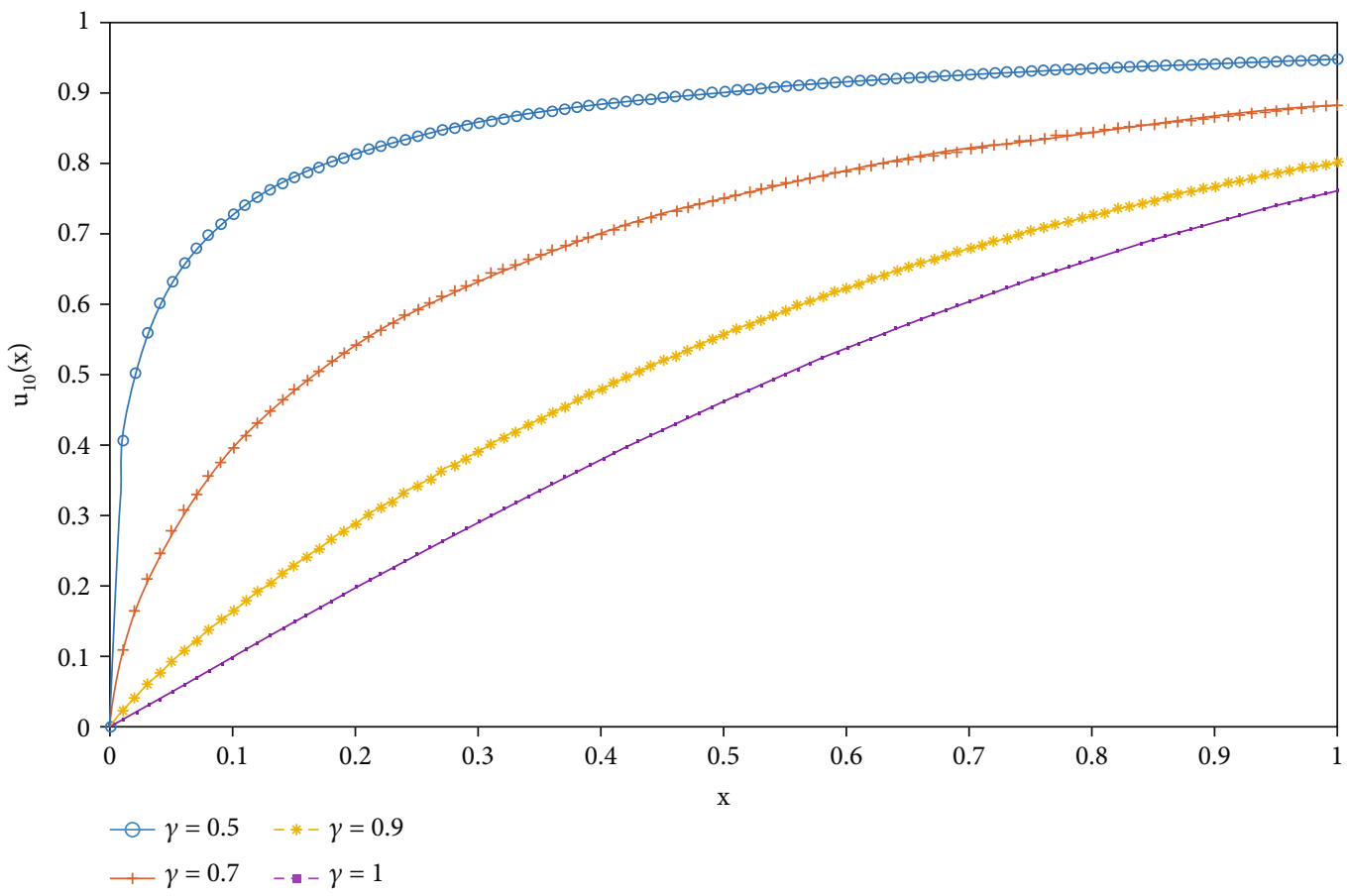

FIGURE 5: The approximate solutions for various values of $\gamma=\alpha$ in Example 23.

This problem has the exact solution $u_{e}(x)=\left[\exp \left(2 x^{\gamma} / \gamma\right)\right.$ $-1] /\left[\exp \left(2 x^{\gamma} / \gamma\right)+1\right]$ (see [21]), and it has been treated by many authors using various methods such as the collocation method, operational matrix method, homotopy perturbation Pade technique, homotopy analysis method, modified homotopy perturbation (MHP) method, modified variational iteration method, B-spline operational matrix method, and polynomial least squares method (see, for example, [54, 55]. In all these methods, the authors used the Caputo fractional derivative.

Now, regarding the proposed method here, the approximated analytical solution of the initial value problem (85) has been computed for $\mathscr{M}=10$ with various values of $\gamma=\alpha$. Figure 5 shows the approximate solutions for $\mathscr{M}=10$ and various values of $\gamma=\alpha=0.5,0.7,0.9,1$. Table 4 illustrates both numerical and exact solutions of (85). The comparison between the obtained values of $u(x)$ by the presented method and the modified Homotopy perturbation method (MHPM) given in [55] for $\gamma=1$ and $\mathscr{M}=10$ is shown in Table 5 . Table 5 illustrates that our method is more accurate compared to other methods.

Example 24. Suppose that a system of fractional differential equations is given in the form

$$
\begin{array}{ll}
\mathscr{D}^{\gamma_{1}} u_{1}(x)=u_{1}(x)+u_{2}(x), & 0<\gamma_{1} \leq 1,0 \leq x \leq 1, \\
\mathscr{D}^{\gamma_{2}} u_{2}(x)=-u_{1}(x)+u_{2}(x), & 0<\gamma_{2} \leq 1,
\end{array}
$$


TABLe 4: Obtained values of $u(x)$ for Example 23 by the present method with $\mathscr{M}=10$ and $\gamma=\alpha=0.7$ and 0.9 .

\begin{tabular}{lcccr}
\hline$x$ & Exact solution $u_{e}(x)$ & & \multicolumn{2}{c}{$\gamma=\alpha=0.9$} \\
\hline 0.1 & 0.277560937258027 & 0.396329287768680 & Exact solution $u_{e}(x)$ & 0.138975357216608 \\
0.2 & 0.432562507032238 & 0.541472216303135 & 0.255255338098812 & 0.165142712549575 \\
0.3 & 0.547648770236210 & 0.634513884465960 & 0.359212661331767 & 0.287926712330121 \\
0.4 & 0.636470555494007 & 0.700774990734457 & 0.451905777263955 & 0.391527449696852 \\
0.5 & 0.706113027676249 & 0.750534511840285 & 0.533789465005938 & 0.480439682175042 \\
0.6 & 0.761214832718165 & 0.789171534868799 & 0.605386508611184 & 0.556914559531437 \\
0.7 & 0.805098130420862 & 0.819896688293229 & 0.667388747653672 & 0.622580109650008 \\
0.8 & 0.840237973205243 & 0.844771155592644 & 0.720626381374415 & 0.678810765325902 \\
0.9 & 0.868514797371047 & 0.865206088424650 & 0.766006660708606 & 0.726831787717051 \\
1 & 0.891373467734719 & 0.882214664339926 & 0.804454800298401 & 0.767744616102944 \\
\hline
\end{tabular}

TABLE 5: Comparison of obtained values of $u_{10}(x)$ with MHPM in Example 23 with $\gamma=1$.

\begin{tabular}{lcccc}
\hline$x$ & MHPM [55] & Present method & Exact solution & Absolute error \\
\hline 0.1 & 0.099668 & 0.099667671416668 & 0.099667994624956 & $3.232082876148868 .10^{-7}$ \\
0.2 & 0.197375 & 0.197375012267825 & 0.197375320224904 & $3.079570785998806 .10^{-7}$ \\
0.3 & 0.291312 & 0.291312318874021 & 0.291312612451591 & $2.935775703234479 .10^{-7}$ \\
0.4 & 0.379944 & 0.379948688365082 & 0.379948962255225 & $2.738901430200881 .10^{-7}$ \\
0.5 & 0.462078 & 0.462116905688459 & 0.462117157260010 & $2.515715507406561 .10^{-7}$ \\
0.6 & 0.536857 & 0.537049339939348 & 0.537049566998035 & $2.270586874674282 .10^{-7}$ \\
0.7 & 0.603631 & 0.604367573599157 & 0.604367777117164 & $2.035180060362447 .10^{-7}$ \\
0.8 & 0.661706 & 0.664036591419464 & 0.664036770267849 & $1.788483853309343 .10^{-7}$ \\
0.9 & 0.709919 & 0.716297714005185 & 0.716297870199024 & $1.561938393935934 .10^{-7}$ \\
1 & 0.746032 & 0.761594024015154 & 0.761594155955765 & $1.319406113975582 .10^{-7}$ \\
\hline
\end{tabular}
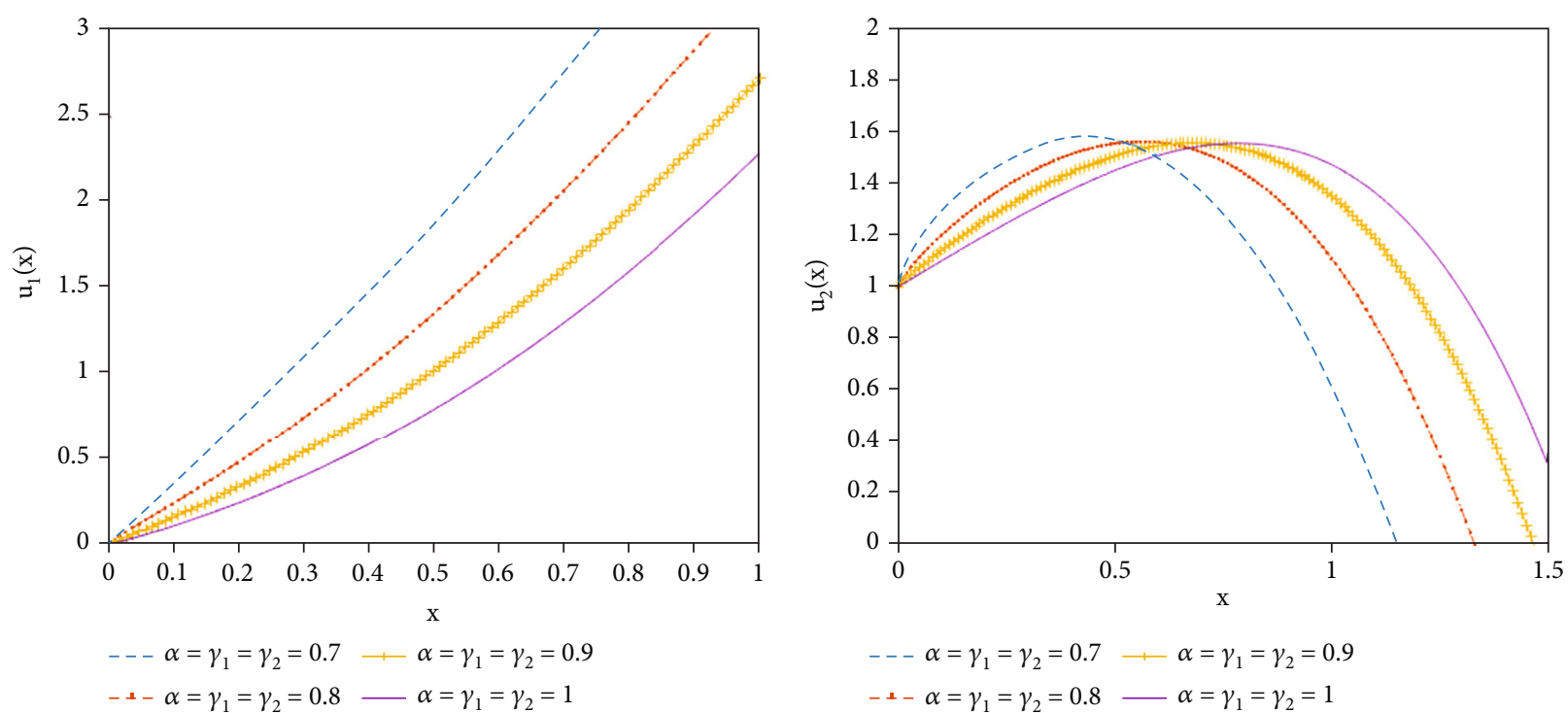

Figure 6: Graph of the approximate solutions $u_{1}(x)$ and $u_{2}(x)$ of Example 24 for $\mathscr{M}=5$ and various values of $\alpha=\gamma_{1}=\gamma_{2}=0.7,0.8,0.9,1$. 
TABLE 6: Absolute errors of $u_{1}(x)$ for various values of $\mathscr{M}=5,8,10$ with $\alpha=\gamma_{1}=\gamma_{2}=1$ in Example 24 .

\begin{tabular}{lccc}
\hline$x$ & $\mathscr{M}=5$ & $\mathscr{M}=8$ & $\mathscr{M}=10$ \\
\hline 0.1 & $2.175152855412623 .10^{-4}$ & $9.162421859512191 .10^{-8}$ & $1.758976284592979 .10^{-10}$ \\
0.2 & $2.563542720906999 .10^{-4}$ & $1.010603222372797 .10^{-7}$ & $1.884819425658612 .10^{-10}$ \\
0.3 & $2.455251579074233 .10^{-4}$ & $1.112064359635733 .10^{-7}$ & $2.046192475246368 .10^{-10}$ \\
0.4 & $2.320713758760705 .10^{-4}$ & $1.218391399387871 .10^{-7}$ & $2.191098474404878 .10^{-10}$ \\
0.5 & $2.201273528237466 .10^{-4}$ & $1.315382035648231 .10^{-7}$ & $2.333426724860480 .10^{-10}$ \\
0.6 & $1.996737905732100 .10^{-4}$ & $1.409308454622984 .10^{-7}$ & $2.471041121886989 .10^{-10}$ \\
0.7 & $1.643238206067162 .10^{-4}$ & $1.489432328394127 .10^{-7}$ & $2.648922750010470 .10^{-10}$ \\
0.8 & $1.175657989381895 .10^{-4}$ & $1.556332410815428 .10^{-7}$ & $2.906084812992896 .10^{-10}$ \\
0.9 & $6.701651870429315 .10^{-5}$ & $1.606609913492092 .10^{-7}$ & $3.310520786806953 .10^{-10}$ \\
1 & $6.404833938994381 .10^{-6}$ & $1.601412220569667 .10^{-7}$ & $4.151750389509799 .10^{-10}$ \\
\hline
\end{tabular}

TABLE 7: Absolute errors of $u_{2}(x)$ for various values of $\mathscr{M}=5,8,10$ with $\alpha=\gamma_{1}=\gamma_{2}=1$ in Example 24 .

\begin{tabular}{lccc}
\hline$x$ & $\mathscr{M}=5$ & $\mathscr{M}=8$ & $\mathscr{M}=10$ \\
\hline 0.1 & $1.764442982358745 .10^{-4}$ & $1.289165400939913 .10^{-8}$ & $6.228629314555091 .10^{-12}$ \\
0.2 & $2.483473612144735 .10^{-4}$ & $3.801071933089472 .10^{-9}$ & $1.613098247747954 .10^{-11}$ \\
0.3 & $2.943918013066388 .10^{-4}$ & $6.996798181222391 .10^{-9}$ & $3.734106142077792 .10^{-11}$ \\
0.4 & $3.463545496320502 .10^{-4}$ & $1.992154729449800 .10^{-8}$ & $6.483630453443810 .10^{-11}$ \\
0.5 & $4.089060754978633 .10^{-4}$ & $3.538677484194269 .10^{-8}$ & $9.820822938741669 .10^{-11}$ \\
0.6 & $4.761667760399912 .10^{-4}$ & $5.339426604651616 .10^{-8}$ & $1.410768556814162 .10^{-10}$ \\
0.7 & $5.435609065997361 .10^{-4}$ & $7.429242006830613 .10^{-8}$ & $2.010920588084222 .10^{-10}$ \\
0.8 & $6.132891968498514 .10^{-4}$ & $9.811361566232743 .10^{-8}$ & $2.860794615286945 .10^{-10}$ \\
0.9 & $6.915106434315280 .10^{-4}$ & $1.250216107427356 .10^{-7}$ & $4.199493848745102 .10^{-10}$ \\
1 & $7.750844548684034 .10^{-4}$ & $1.556557391707215 .10^{-7}$ & $6.201785524511703 .10^{-10}$ \\
\hline
\end{tabular}

with the primary conditions

$$
u_{1}(0)=0, u_{2}(0)=1
$$

We proceed the solution as follows. When $\gamma_{1}=\gamma_{2}=1$, the exact solution of this system was given in $[56,57]$ as $u_{1}$ $(x)=e^{x} \sin x$, and $u_{2}(x)=e^{x} \cos x$. Applying the same technique described above and by putting $\mathscr{M}=5$ in (71) with $\alpha$ $=\gamma_{1}=\gamma_{2}$, we have computed the approximate solution for the values $\alpha=\gamma_{1}=\gamma_{1}=0.7,0.8,0.9,1$. Figure 6 shows the graphs of approximate solution for various values of $\alpha=\gamma_{1}$ $=\gamma_{2}$ with $\mathscr{M}=5$. Tables 6 and 7 display the corresponding absolute error to our approximate solutions for $\alpha=\gamma_{1}=\gamma_{2}$ $=1$ and various values of $\mathscr{M}$. Note that the absolute error tends to zero when the terms $\mathscr{M}$ of CFBFs increase.

\section{Conclusion}

Exploring the multifaceted applications of Bessel functions in several fields of science with recognition of the growing impact conformable fractional calculus has in many applications, this paper exhibits further developments on the conformable fractional-order Bessel functions (CFBFs). The novelty of this research paper is determined by explaining the comparison between our obtained solutions (results) and the solutions in previously published articles by various authors. Such novelty may be described as follows. In the first part of the paper, some useful formulas concerning the properties of conformable fractional Laplace transforms are obtained. These formulas are successfully employed to obtain the solutions for a new type of fractional kinetic equations associated with the CFBFs in the conformable fractional sense which is generalized and developed in this study. These solutions are newly presented compared with those given by various authors (see [38, 58-61]).

In addition, an interesting orthogonal relation of the CFBFs is established. This gives rise to the discussion of functions expansions where we present a given function in a series of the CFBFs. Consequently, we investigate the analytical and approximate solutions of some linear and nonlinear CFDEs using a proposed scheme depending on the collocation method, involving CFBFs. Particular emphasis is paid to indicate that our approach, in some sense, is easily applicable and provides more accurate results with refined errors. The proposed scheme is used to approximate the solutions of linear, nonlinear CFDEs, and also systems of CFDEs. The numerical 
examples show the validity and efficiency of the method. Notably, it is demonstrated that the presented method works well and achieves better accuracy compared with exact solutions and with results obtained using other methods. Our scheme is beneficial in the way that using the fractional-order Bessel functions leads to more accurate results in approximating the fractional function. Because of this characteristic, FBFs are more effective than Bessel functions in treating fractional problems. Because Bessel polynomials have smaller coefficients than Chebyshev, Legendre, and Bernoulli polynomials, the computational error in the current approach is less. Importantly, the employment of the collocation method provides convenient and accurate solutions. Furthermore, all the given solutions are obtained differently, unlike those established by the authors in [47-55] and the references therein where other fractional derivatives such as Caputo derivative were used.

\section{Data Availability}

The data used to support the findings of this study are included within the article.

\section{Conflicts of Interest}

The authors declare there is no conflict of interest.

\section{Authors' Contributions}

The authors have contributed equally to this work.

\section{Acknowledgments}

The authors extend their appreciation to the Deanship of Scientific Research at King Khalid University, Saudi Arabia, through research group program under grant R.G.P.1/86/42.

\section{References}

[1] S. Qureshi, "Effects of vaccination on measles dynamics under fractional conformable derivative with Liouville-Caputo operator," The European Physical Journal Plus, vol. 135, no. 1, 2020.

[2] M. Dalir and M. Bashour, "Applications of fractional calculus," Applied Mathematical Sciences, vol. 4, no. 21, pp. 1021-1032, 2010.

[3] A. A. Kilbas, H. M. Srivastava, and J. J. Trujillo, Theory and Applications of Fractional Differential Equations, (Vol. 204), Elsevier, 2006.

[4] I. Podlubny, Fractional Differential Equations: An Introduction to Fractional Derivatives, Fractional Differential Equations, to Methods of Their Solution and Some of Their Applications, Elsevier, 1998.

[5] G. Montseny, J. Audounet, and B. Mbodje, "Optimal models of fractional integrators and application to systems with fading memory," in Proceedings of IEEE Systems Man and Cybernetics Conference-SMC, Le Touquet, France, 1993.

[6] T. Kaczorek and K. Rogowski, Fractional Linear Systems and Electrical Circuits, Springer International Publishing, Cham, Switzerland, 2015.

[7] F. Mirzaee and N. Samadyar, "On the numerical method for solving a system of nonlinear fractional ordinary differential equations arising in HIV infection of CD4 T cells," Iranian
Journal of Science and Technology, Transactions A: Science, vol. 43, no. 3, pp. 1127-1138, 2019.

[8] Q. M. Al-Mdallal, "On fractional-Legendre spectral Galerkin method for fractional Sturm-Liouville problems," Chaos, Solitons and Fractals, vol. 116, pp. 261-267, 2018.

[9] Q. M. Al-Mdallal, H. Yusuf, and A. Ali, “A novel algorithm for time-fractional foam drainage equation," Alexandria Engineering Journal, vol. 59, no. 3, pp. 1607-1612, 2020.

[10] K. S. Miller and B. Ross, An Introduction to the Fractional calculus and Fractional Differential Equations, Wiley, 1993.

[11] M. F. Simões Patrício, H. Ramos, and M. Patrício, "Solving initial and boundary value problems of fractional ordinary differential equations by using collocation and fractional powers," Journal of Computational and Applied Mathematics, vol. 354, pp. 348-359, 2019.

[12] R. Khalil, M. Al Horani, A. Yousef, and M. Sababheh, "A new definition of fractional derivative," Journal of Computational and Applied Mathematics, vol. 264, pp. 65-70, 2014.

[13] V. E. Tarasov, "No violation of the Leibniz rule. No fractional derivative," Communications in Nonlinear Science and Numerical Simulation, vol. 18, no. 11, pp. 2945-2948, 2013.

[14] M. Abul-Ez, M. Zayed, A. Youssef, and M. De la Sen, "On conformable fractional Legendre polynomials and their convergence properties with applications," Alexandria Engineering Journal, vol. 59, no. 6, pp. 5231-5245, 2020.

[15] M. Abul-Ez, M. Zayed, and A. Youssef, "Further study on the conformable fractional Gauss hypergeometric function," Aims Mathematics, vol. 6, no. 9, pp. 10130-10163, 2021.

[16] T. Abdeljawad, "On conformable fractional calculus," Journal of Computational and Applied Mathematics, vol. 279, pp. 5766, 2015.

[17] D. R. Anderson, E. Camrud, and D. J. Ulness, "On the nature of the conformable derivative and its applications to physics," Journal of Fractional Calculus and Applications, vol. 10, no. 2, pp. 92-135, 2019.

[18] H. W. Zhou, S. Yang, and S. Q. Zhang, "Conformable derivative approach to anomalous diffusion," Physica A: Statistical Mechanics and its Applications, vol. 491, pp. 1001-1013, 2018.

[19] A. Atangana, D. Baleanu, and A. Alsaedi, "New properties of conformable derivative," Open Mathematics, vol. 13, no. 1, pp. 889-898, 2015.

[20] O. T. Birgani, S. Chandok, N. Dedovic, and S. Radenovic, "A note on some recent results of the conformable fractional derivative," Advances in the Theory of Nonlinear Analysis and its Application, vol. 3, no. 1, pp. 11-17, 2018.

[21] E. Ünal and A. Gökdoğan, "Solution of conformable fractional ordinary differential equations via differential transform method," Optik, vol. 128, pp. 264-273, 2017.

[22] D. Zhao and M. Luo, "General conformable fractional derivative and its physical interpretation," Calcolo, vol. 54, no. 3, pp. 903-917, 2017.

[23] A. Younas, T. Abdeljawad, R. Batool, A. Zehra, and M. A. Alqudah, "Linear conformable differential system and its controllability," Advances in Difference Equations, vol. 2020, no. 1, 26 pages, 2020.

[24] A. Atangana and D. Baleanu, "New fractional derivatives with nonlocal and non-singular kernel: theory and application to heat transfer model," Thermal Science, vol. 20, no. 2, pp. 763$769,2016$.

[25] J. Niedziela, Bessel Functions and Their Applications, University of Tennessee, Knoxville, 2008. 
[26] A. Gökdoğan, E. Ünal, and E. Çelik, "Conformable fractional Bessel equation and Bessel functions," 2015, https://arxiv.org/ abs/1506.07382.

[27] H. Dehestani, Y. Ordokhani, and M. Razzaghi, "Fractionalorder Bessel functions with various applications," Applications of Mathematics, vol. 64, no. 6, pp. 637-662, 2019.

[28] M. Izadi and C. Cattani, "Generalized Bessel polynomial for multi-order fractional differential equations," Symmetry, vol. 12 , no. 8, 2020.

[29] M. A. Abul-Ez, "Bessel polynomial expansions in spaces of holomorphic functions," Journal of Mathematical Analysis and Applications, vol. 221, no. 1, pp. 177-190, 1998.

[30] M. Abdalla, M. Abul-Ez, and J. Morais, "On the construction of generalized monogenic Bessel polynomials," Mathematical Methods in the Applied Sciences, vol. 41, no. 18, pp. 93359348, 2018.

[31] Ş. Yüzbaşi, N. Şahin, and M. Sezer, "Numerical solutions of systems of linear Fredholm integro-differential equations with Bessel polynomial bases," Computers \& Mathematics with Applications, vol. 61, no. 10, pp. 3079-3096, 2011.

[32] K. Parand, M. Nikarya, and J. A. Rad, "Solving non-linear lane-Emden type equations using Bessel orthogonal functions collocation method," Celestial Mechanics and Dynamical Astronomy, vol. 116, no. 1, pp. 97-107, 2013.

[33] E. Tohidi and H. Saberi Nik, "A Bessel collocation method for solving fractional optimal control problems," Applied Mathematical Modelling, vol. 39, no. 2, pp. 455-465, 2015.

[34] E. Grosswald, Bessel Polynomials, Springer-Verlag, Berlin Heidelberg, 1978.

[35] D. E. Rainville, Special Functions, Chelsea, New York, 1960.

[36] A. Wiman, "Über den fundamentalsatz in der teorie der funktionen $E_{a}(x)$," Acta Mathematica, vol. 29, pp. 191-201, 1905.

[37] H. J. Haubold and A. M. Mathai, "The fractional kinetic equation and thermonuclear functions," Astrophysics and Space Science, vol. 273, no. 1/4, pp. 53-63, 2000.

[38] R. K. Saxena and S. L. Kalla, "On the solutions of certain fractional kinetic equations," Applied Mathematics and Computation, vol. 199, no. 2, pp. 504-511, 2008.

[39] S. G. Samko, A. A. Kilbas, and O. I. Marichev, Fractional Integrals and Derivatives, Yverdon-les-Bains, Gordon and Breach Science Publishers, Yverdon, Switzerland, 1993.

[40] W. W. Bell, Special Functions for Scientists and Engineers, Courier Corporation, 2004.

[41] R. P. Boas and R. C. Buck, Polynomial Expansions of Analytic Functions, Springer-Verlag, Berlin Heidelberg, 1958.

[42] G. Faber, "Über polynomische Entwickelungen," Mathematische Annalen, vol. 57, no. 3, pp. 389-408, 1903.

[43] J. Whittaker and C. Gattegno, Sur les Séries de Base de Polynbmes Quelconques, Gauthier-Villars, Paris, 1949.

[44] M. A. Abul-ez and D. Constales, "Basic sets of pofynomials in Clifford analysis," Complex Variables, Theory and Application: An International Journal, vol. 14, no. 1-4, pp. 177-185, 1990.

[45] M. Zayed, M. Abul-Ez, and J. P. Morais, "Generalized derivative and primitive of Cliffordian bases of polynomials constructed through Appell monomials," Computational Methods and Function Theory, vol. 12, no. 2, pp. 501-515, 2012.

[46] M. Zayed, "Generalized Hadamard product bases of special monogenic polynomials," Advances in Applied Clifford Algebras, vol. 30, no. 1, 2020.
[47] S. Kazem, S. Abbasbandy, and S. Kumar, "Fractional-order Legendre functions for solving fractional-order differential equations," Applied Mathematical Modelling, vol. 37, no. 7, pp. 5498-5510, 2013.

[48] H. Jafari, S. Das, and H. Tajadodi, "Solving a multi-order fractional differential equation using homotopy analysis method," Journal of King Saud University-Science, vol. 23, no. 2, pp. 151155, 2011.

[49] A. Saadatmandi and M. Dehghan, "A new operational matrix for solving fractional-order differential equations," Computers and Mathematics with Applications, vol. 59, no. 3, pp. 13261336, 2010.

[50] N. H. Sweilam, M. M. Khader, and R. F. Al-Bar, "Numerical studies for a multi-order fractional differential equation," Physics Letters A, vol. 371, no. 1-2, pp. 26-33, 2007.

[51] H. Çerdik Yaslan and F. Mutlu, "Numerical solution of the conformable differential equations via shifted Legendre polynomials," International Journal of Computer Mathematics, vol. 97, no. 5, pp. 1016-1028, 2020.

[52] M. M. Khader, T. S. El Danaf, and A. S. Hendy, "Efficient spectral collocation method for solving multi-term fractional differential equations based on the generalized Laguerre polynomials," Journal of Fractional Calculus and Applications, vol. 3, pp. 1-14, 2012.

[53] K. Parand and M. Nikarya, "Application of Bessel functions for solving differential and integro- differential equations of the fractional order," Applied Mathematical Modelling, vol. 38, no. 15-16, pp. 4137-4147, 2014.

[54] C. Bota and B. Căruntu, "Analytical approximate solutions for quadratic Riccati differential equation of fractional order using the polynomial least squares method," Chaos, Solitons and Fractals, vol. 102, pp. 339-345, 2017.

[55] Z. Odibat and S. Momani, "Modified homotopy perturbation method: application to quadratic Riccati differential equation of fractional order," Chaos, Solitons \& Fractals, vol. 36, no. 1, pp. 167-174, 2008.

[56] M. Zurigat, S. Momani, Z. Odibat, and A. Alawneh, "The homotopy analysis method for handling systems of fractional differential equations," Applied Mathematical Modelling, vol. 34, no. 1, pp. 24-35, 2010.

[57] S. Momani and Z. Odibat, "Numerical approach to differential equations of fractional order," Journal of Computational and Applied Mathematics, vol. 207, no. 1, pp. 96-110, 2007.

[58] D. Kumar, S. D. Purohit, A. Secer, and A. Atangana, "On generalized fractional kinetic equations involving generalized Bessel function of the first kind," Mathematical Problems in Engineering, vol. 2015, Article ID 289387, 7 pages, 2015.

[59] P. Agarwal, M. Chand, D. Baleanu, D. O’Regan, and S. Jain, "On the solutions of certain fractional kinetic equations involving k-Mittag-Leffler function," Advances in Difference Equations, vol. 2018, no. 1, 2018.

[60] G. Singh, P. Agarwal, M. Chand, and S. Jain, "Certain fractional kinetic equations involving generalized k-Bessel function," Transactions of A. Razmadze Mathematical Institute, vol. 172, no. 3, pp. 559-570, 2018.

[61] P. Agarwal, M. Chand, and G. Singh, "Certain fractional kinetic equations involving the product of generalized kBessel function," Alexandria Engineering Journal, vol. 55, no. 4, pp. 3053-3059, 2016. 